\title{
Prefrontal Neurons Represent Motion Signals from Across the Visual Field But for Memory-Guided Comparisons Depend on Neurons Providing These Signals
}

\author{
ㅈous Wimmer, ${ }^{1}$ Philip Spinelli, ${ }^{2}$ and ${ }^{\circledR}$ Tatiana Pasternak ${ }^{2}$ \\ ${ }^{1}$ Institut d'Investigacions Biomèdiques August Pi i Sunyer 08036 Barcelona, Spain, and ${ }^{2}$ Department of Neuroscience and Center for Visual Science, \\ University of Rochester, Rochester, New York 14642
}

Visual decisions often involve comparisons of sequential stimuli that can appear at any location in the visual field. The lateral prefrontal cortex (LPFC) in nonhuman primates, shown to play an important role in such comparisons, receives information about contralateral stimuli directly from sensory neurons in the same hemisphere, and about ipsilateral stimuli indirectly from neurons in the opposite hemisphere. This asymmetry of sensory inputs into the LPFC poses the question of whether and how its neurons incorporate sensory information arriving from the two hemispheres during memory-guided comparisons of visual motion. We found that, although responses of individual LPFC neurons to contralateral stimuli were stronger and emerged 40 ms earlier, they carried remarkably similar signals about motion direction in the two hemifields, with comparable direction selectivity and similar direction preferences. This similarity was also apparent around the time of the comparison between the current and remembered stimulus because both ipsilateral and contralateral responses showed similar signals reflecting the remembered direction. However, despite availability in the LPFC of motion information from across the visual field, these "comparison effects" required for the comparison stimuli to appear at the same retinal location. This strict dependence on spatial overlap of the comparison stimuli suggests participation of neurons with localized receptive fields in the comparison process. These results suggest that while LPFC incorporates many key aspects of the information arriving from sensory neurons residing in opposite hemispheres, it continues relying on the interactions with these neurons at the time of generating signals leading to successful perceptual decisions.

Key words: direction selectivity; hemifields; working memory

Significance Statement

Visual decisions often involve comparisons of sequential visual motion that can appear at any location in the visual field. We show that during such comparisons, the lateral prefrontal cortex (LPFC) contains accurate representation of visual motion from across the visual field, supplied by motion processing neurons. However, at the time of comparison, LPFC neurons can only use this information to compute the differences between the stimuli, if stimuli appear at the same retinal location, implicating neurons with localized receptive fields in the comparison process. These findings show that sensory comparisons rely on the interactions between LPFC and sensory neurons that not only supply sensory signals but also actively participate in the comparison of these signals at the time of the decision.

\section{Introduction}

Comparing two visual stimuli that occur at different times demands the coordination of bottom-up and top-down pro-

\footnotetext{
Received March 14, 2016; revised June 24, 2016; accepted July 19, 2016.

Author contributions: T.P. designed research; K.W., P.S., and T.P. performed research; K.W. and T.P. analyzed data; K.W. and T.P. wrote the paper.

This work was supported by the National Eye Institute Grant R01 EY11749 to T.P. and Grant P30 EY01319 to the Center for Visual Science. We thank Aaron Ketting-Olivier for help with the data analysis; Kostas Michalopoulos and Ping Ren participated in some of the data collection and in early stages of data analysis; Martin Gira and Gina DeMeo for technical assistance; Albert Compte for useful suggestions; and Larry Abbott, Ben Hayden, and Ruben MorenoBote for comments on the manuscript.
}

cesses. Such tasks require processing and storage of sensory stimuli, followed by their retrieval and comparison leading to perceptual decision. It is widely accepted that the prefrontal cortex (PFC) integrates the various components of such tasks in its neural activity (Freedman et al., 2001; Fuster, 2008; Tanji and Hoshi, 2008; D'Esposito and Postle, 2015). It is recipro-

The authors declare no competing financial interests.

Correspondence should be addressed to Dr. Tatiana Pasternak, Department of Neuroscience, Box 603, University of Rochester, Rochester, NY 14642. E-mail: tania@cvs.rochester.edu.

DOI:10.1523/JNEUROSCI.0843-16.2016

Copyright $\odot 2016$ the authors $\quad 0270-6474 / 16 / 369351-14 \$ 15.00 / 0$ 
cally connected with neurons processing sensory information and is thought to be an important source of top-down influences it exerts on these neurons. When such tasks involve comparisons of motion directions presented at the fovea, neurons in the lateral PFC (LPFC) show direction-selective (DS) responses that resemble responses in the motion processing area MT, indicative of their bottom-up origin (Zaksas and Pasternak, 2006; Hussar and Pasternak, 2009, 2012). Indeed, MT is a likely source of directional signals as it provides direct inputs to the LPFC in the same hemisphere (Jacobson and Trojanowski, 1977; Barbas, 1988; Schall et al., 1995; Petrides and Pandya, 2006; Ninomiya et al., 2012). However, because of strict retinotopy and localized receptive fields representing nearly exclusively the contralateral visual field, direct inputs from MT are likely to only supply the information about contralateral stimuli. Given that prefrontal neurons also respond to visual stimuli presented in the ipsilateral hemifield (e.g., Boch and Goldberg, 1989; Funahashi et al., 1989; Sakagami and Niki, 1994; Rainer et al., 1998; Rainer et al., 1999; Everling et al., 2002; Kadohisa et al., 2013; Lennert and MartinezTrujillo, 2013; Kadohisa et al., 2015), visual information must also reach LPFC indirectly, most likely by way of strong callosal connections from the opposite LPFC (see Fig. 1A) (Schwartz and Goldman-Rakic, 1984; but see Adachi et al., 2012). The retinotopic nature of sensory inputs to the LPFC poses a challenge to the mechanisms underlying the top-down influences this region provides to upstream sensory neurons. While there is compelling evidence that these top-down influences are aimed primarily at visual cortical areas within the same hemisphere (Barceló et al., 2000; Gregoriou et al., 2014; Pasternak et al., 2015), little is known whether LPFC also interacts with sensory neurons residing in the opposite hemisphere. An important first step in addressing this problem is to determine whether and how LPFC neurons incorporate and use sensory information arriving from the opposite hemisphere into signals it provides to other components of the circuitry underlying sensory comparison tasks.

We compared responses recorded in the LPFC during comparisons of directions of two sequential stimuli presented in the contralateral or the ipsilateral hemifields. During the first stimulus, responses to the contralateral motion were stronger and occurred $\sim 40 \mathrm{~ms}$ earlier, reflecting the dominance of signals that reach LPFC directly from motion processing neurons residing in the same hemisphere. However, during the second stimulus, near the time of the comparison and decision, ipsilateral responses were enhanced and the contralateral dominance largely vanished, highlighting the importance of task demands in the representation of motion signals in the LPFC (Hussar and Pasternak, 2009). The similarity in responses to ipsilateral and contralateral stimuli was also reflected in their selectivity for motion direction and was apparent in the response to the second stimulus that carried signals reflecting the difference between the current and the preceding stimulus. However, these comparison signals nearly disappeared when the two stimuli were spatially separated, suggesting that their generation requires the participation of neurons with localized receptive fields. Thus, during comparisons of motion directions, the LPFC neurons seamlessly incorporate the information about visual motion arriving from sensory neurons in both hemispheres. However, our results suggest the process of comparison reflected in the activity of these neurons depends on signals supplied by the upstream sensory neurons.

\section{Materials and Methods}

\section{Subjects}

We recorded from the LPFC of three adult male rhesus macaque monkeys. All experiments were performed in accordance with the guidelines published in the National Institutes of Health Guide for the care and use of laboratory animals and approved for use by the University of Rochester Committee for Animal Research.

\section{Behavioral task}

The structure of the behavioral task is outlined in Figure 1A. During the task, the animals viewed two sequential stimuli, S1 and S2, separated by a brief delay. For two of the monkeys, the delay was set to $1500 \mathrm{~ms}$ (M908 and M201), whereas for the third animal, the delay was shorter, ranging between $500 \mathrm{~ms}$ and $1000 \mathrm{~ms}$ (M611). Stimuli appeared at one of the four quadrants of the ipsilateral or the contralateral visual field, with an equal distance from the fixation spot, at $7^{\circ}$ eccentricity. In the standard version of the task, S1 and S2 appeared at the same position. In a subset of recording, sessions S1 and S2 were spatially separated, with S2 appearing in the contralateral hemifield and $\mathrm{S} 1$ either placed in the opposite, ipsilateral, hemifield (remote-ipsi; see Fig. $8 A$ ) or in the same, contralateral, hemifield but with a vertical displacement relative to S2 (remote-contra; see Fig. $8 B$ ). In all conditions, S1 and S2 always had the same distance from the fixation point. Both S1 and S2 moved in one of eight directions $\left(0^{\circ}, 45^{\circ}, 90^{\circ}, 135^{\circ}, 180^{\circ}, 225^{\circ}, 270^{\circ}\right.$, and $\left.315^{\circ}\right)$ and on each trial the directions of S1 and S2 were either identical (Same trial) or different (Different trials). The direction difference was usually $90^{\circ}$ for M908 and M201 and $180^{\circ}$ for M611. Each trial began with the presentation of a small target the animals were required to fixate $\left( \pm 1.5^{\circ}\right)$ throughout its duration. The period of fixation preceding the onset of S1 was set to 1000 $\mathrm{ms}$, and each stimulus lasted $500 \mathrm{~ms}$. After the offset of S2 and additional $1000 \mathrm{~ms}$, the fixation target was replaced with two small circular targets on either side of the display, and the animals were rewarded for making a saccade to the right target to report same direction trial and for making a saccade to the left target to report different direction trial. All directions were presented with equal probability during both S1 and S2. During each recording session, the tasks with stimuli in the contralateral and ipsilateral quadrants were run in blocks of typically 128 trials ( 16 trials for each stimulus direction).

\section{Visual stimuli and fixation control}

The random-dot stimuli were displayed on a 19 inch monitor (Ilyama Vision Master Pro 513, $75 \mathrm{~Hz}$ refresh rate) at a distance of $57 \mathrm{~cm}$ from the animals. The dot density was set to $4.7 \mathrm{dots} / \mathrm{deg}^{2}$, with each dot subtending $0.03^{\circ}$ of visual angle, at the luminance of $15 \mathrm{~cd} / \mathrm{m}^{2}$. Each dot persisted for the entire stimulus duration. The stimuli were $5^{\circ}$ in diameter, moving coherently at $10^{\circ} / \mathrm{s}$ to $15^{\circ} \%$. They were presented at $7^{\circ}$ eccentricity in the upper or lower quadrants of the ipsilateral or contralateral hemifields. Within each session, the order of testing in the contralateral and ipsilateral hemifield was irregular. Eye position was monitored with an infrared video eye-tracking package (ISCAN), and monkeys were required to maintain fixation within $1.5^{\circ}$ of a centrally presented fixation target in all trials.

\section{Physiological recordings}

Recording locations were identified from structural MRI scans obtained with a 3T scanner. Recording chambers were placed over the right LPFC in Monkeys M201 and M908, and on both sides in Monkey M611. The recordings were performed with single tungsten glass-coated microelectrodes (0.5-3 M $\Omega$ at $f=1 \mathrm{kHz}$; Alpha Omega Engineering) in Monkeys M611 and M908. For Monkey M201, we also used linear electrodes with 16 recording sites separated by 100 or $150 \mu \mathrm{m}$ (U-probes and V-probes; Plexon). Electrodes were positioned over the chamber-enclosed craniotomy using a CILUX grid with $1 \mathrm{~mm}$ spaced openings (Crist Instruments). Custom-made steel guide tubes were inserted into the hole chosen for each day's recording to provide structural stability. Guide tubes were lowered to the dura but did not penetrate it. Electrodes were driven through the dura using a NAN electrode drive (NAN Instruments). To maximize recording stability, data collection began 30-60 min after the placement of electrodes. Recordings were obtained with 
Plexon Multichannel Acquisition Processor systems working concomitantly with the Windows-based real-time data acquisition system TEMPO (Reflective Computing). Activity of each neuron used in the analysis was recorded with S1 and S2 appearing in contralateral and ipsilateral visual field in separate blocks of trial.

\section{Data analysis}

All spike sorting was done manually after the experiment using Offline Sorter (version 3.3.2; Plexon), based on principal component analysis of the spike waveforms. Activity recorded with linear electrodes from M201 included both single-unit and multiunit activity. All further data analyses were carried out in MATLAB (The MathWorks).

Responses to visual motion. The activity of each neuron during S1 and S2 was analyzed to determine whether it responded to stimuli appearing during S1 and S2. Spike counts were computed for five nonoverlapping $100 \mathrm{~ms}$ windows during the $S 1$ period $(500 \mathrm{~ms})$, separately for trials with each of the eight stimulus directions. A neuron was classified as responsive if at least $100 \mathrm{~ms}$ of activity were significantly different from baseline for at least one direction ( $p<0.00125$, Wilcoxon tests; Bonferroni corrected). Baseline was defined as the firing rate during $300 \mathrm{~ms}$ before the onset of S1. Responsive neurons were classified as excitatory or suppressive depending on whether the mean activity during the stimulus presentation (50-550 ms from stimulus onset) was greater or less than baseline, respectively. Responses were then computed in sliding $100 \mathrm{~ms}$ spike count windows with $10 \mathrm{~ms}$ steps, and the baseline activity was subtracted before further analysis. The firing rates of the excitatory neurons (see Fig. $3 A$ ) were obtained from responses to the stimulus direction that elicited the highest average response, whereas the firing rates of the suppressive neurons (see Fig. $3 B$ ) were obtained from responses to the stimulus direction that elicited the lowest average response. For both suppressive and excitatory neurons, these "best" direction responses were maximally different from baseline activity. The best stimulus directions were chosen independently for contralateral and ipsilateral stimuli and for S1 and S2.

Response latency. For computing response latencies, responses for each neuron were computed for sliding $100 \mathrm{~ms}$ windows in $5 \mathrm{~ms}$ steps, and the baseline activity in a $300 \mathrm{~ms}$ window before the stimulus onset was subtracted. The response latency was defined as the time during the stimulus at which the response exceeded $75 \%$ of the maximum response (for excitatory neurons) or $75 \%$ of the minimum response (for suppressive neurons). This yields latencies that do not depend on response strength, unlike other measures, such as the time point where the response becomes significantly different from baseline activity. This is important because we compared the latencies for contralateral and ipsilateral responses that also differed in their firing rates.

Response change index (RCI). To compare the responses during S1 and $\mathrm{S} 2$, we computed for each neuron a RCI $=\left(r_{\text {peak }}^{\mathrm{S2}}-r_{\text {peak }}^{\mathrm{S1}}\right) /$ $\left(r_{\text {peak }}^{\mathrm{S2}}+r_{\text {peak }}^{\mathrm{S1}}\right)$, where $r_{\text {peak }}^{\mathrm{S1}}$ and $r_{\text {peak }}^{\mathrm{S2}}$ for excitatory neurons are the peak responses during S1 and S2, respectively (obtained from the $100 \mathrm{~ms}$ window with the maximum response during the interval from 50 to $550 \mathrm{~ms}$ from onset of the corresponding stimulus). For suppressive neurons, the peak responses were defined as the minimum firing rates. The RCI was computed separately for contralateral and ipsilateral stimuli.

Location effect index (LEI). To compare the responses to contralateral and ipsilateral motion stimuli, we computed for each neuron a LEI $=$ $\left(r_{\text {peak }}^{\text {contra }}-r_{\text {peak }}^{\text {ipsi }}\right) /\left(r_{\text {peak }}^{\text {contra }}+r_{\text {peak }}^{\text {ipsi }}\right)$, where $r_{\text {peak }}^{\text {contra }}$ and $r_{\text {peak }}^{\text {ispi }}$ are the peak responses for contralateral and ipsilateral stimuli, respectively. The LEI was computed separately for S1 and S2 stimuli.

Direction selectivity. On each trial, the animals discriminated between pairs of possible eight directions, allowing a relatively precise estimate of direction selectivity of each neuron. The preferred direction $\theta_{\text {pref }}$ of each neuron was determined by computing the circular mean of the eight stimulus directions $\theta_{j}\left(0^{\circ}-315^{\circ}\right.$, in steps of $\left.45^{\circ}\right)$ weighted by the neuron's mean spike count $r_{j}$ over the stimulus period for each direction: $\theta_{\text {pref }}=\arctan \left(\sum_{j=1}^{8} r_{j} \sin \left(\theta_{j}\right) / \sum_{j=1}^{8} r_{j} \cos \left(\theta_{j}\right)\right)$. The antipreferred direction was determined as the one moving to the opposite direction. DS activity was then assessed by comparing the $\mathrm{S} 1$ responses on trials with motion in the preferred and anti-preferred directions. For each neuron, we compiled a receiver operating characteristic (ROC) and computed the area under the ROC (AROC) as a measure of selectivity (Britten et al., 1992). The AROC corresponds to the probability that an ideal observer correctly predicts the stimulus direction given a sample from the set of responses to the preferred and antipreferred directions. AROC values range between 0 and 1 , where $A R O C=0.5$ indicated activity that did not systematically differ between trials, whereas AROC $>0.5$ indicated higher activity for one direction and AROC $<0.5$ indicated higher activity for the other direction. Significance of AROCs was evaluated using permutation tests, based on 1000 shuffles of trials associated with each of the two directions. AROC values for each neuron were computed for spike counts in sliding $100 \mathrm{~ms}$ windows in $10 \mathrm{~ms}$ steps during the period of 50 to $600 \mathrm{~ms}$ after S1 onset, and neurons were considered as significantly DS if they had at least three consecutive time bins of significant activity (AROC $\neq 0.5$ with $p<$ 0.05 , permutation test). The beginning of the DS epoch was marked as the center of the first significant bin. Maximum direction selectivity for each neuron was computed as the highest value obtained by averaging the AROC values over 10 consecutive time bins.

Tuning for motion direction. To estimate the direction tuning curves, we averaged the responses during S1 (from 50 to $600 \mathrm{~ms}$ ) to each of the 8 directions used during the task and subtracted the minimum average response for the tested stimulus directions. For each neuron, we then fitted these average responses with a Gaussian function with three parameters: maximum height, preferred direction, and width. Only neurons that were well fit by the Gaussian function $\left(R^{2}>0.5\right)$ were used for further analysis. The FWHH of the fitted tuning curve was taken as the tuning width of each neuron for ipsilateral and contralateral stimuli. To compute the population tuning curves (see Fig. $6 C$ ), we aligned individual tuning curves by their preferred direction, normalized the responses for each neuron between 0 and 1 , and then averaged across neurons.

Comparison effects (CEs). The direction discrimination task (see Fig. $1 A$ ) consisted of two types of randomly interleaved trials: in half of the trials, S1 and S2 moved in the same direction (S-trials), and in the other half they moved in different directions (D-trials). To examine whether the responses during and after $\mathrm{S} 2$ were affected by the preceding S1, we compared the activity in trials with the same stimulus presented during S2 on S- and D-trials. We called the differences in activity during the two types of trials a CE because they likely reflected the sensory comparison process required by the tasks. To rule out the effects of stimulus selectivity during S2 on CEs, we $z$-scored the spike counts recorded for each stimulus direction. Differences between S-trials and D-trials were evaluated with ROC analysis, using sliding $200 \mathrm{~ms}$ windows in $10 \mathrm{~ms}$ steps. Only neurons that were responsive during S2 were used in this analysis ( $N=152$ neurons). Neurons with AROC values $>0.5$ showed higher activity in S-trials than in D-trials, whereas sites with AROC $<0.5$ showed higher activity in D-trials. Neurons were categorized as same $>$ different $(S>D)$, if they had at least five consecutive time bins of significant $S>D$ activity (AROC $>0.5$ with $p<0.05$, permutation test) in the interval from 0.05 to $1.45 \mathrm{~s}$ after the onset of S2. Similarly, neurons were categorized as different $>$ same $(D>S)$, if they had at least five consecutive time bins of significant D $>S$ activity (AROC $<0.5$ with $p<0.05$, permutation test) in the interval from $0.05 \mathrm{~s}$ to $1.45 \mathrm{~s}$ after the onset of $\mathrm{S} 2$. The onset of the comparison selective epoch was marked as the center of the first significant time bin. If a site showed both $\mathrm{S}>\mathrm{D}$ and $\mathrm{D}>\mathrm{S}$ activity, the effect with the longest duration determined the categorization. Sites that did not meet these criteria were categorized as nonselective. For an easier comparison of the magnitude and the time course of $\mathrm{S}>\mathrm{D}$ and $\mathrm{D}>\mathrm{S}$ effects, neurons with peak AROC modulation $<0.5$ were reflected $\sim 0.5$ (i.e., were replaced by $1-$ AROC). For each neuron, the magnitude of the $\mathrm{CE}$ was computed as the average over AROC values in the $300 \mathrm{~ms}$ window with the highest effect.

\section{Results}

We compared the representation of direct and indirect visual motion signals by evaluating the activity of 197 neurons recorded from three monkeys, while they compared the directions of two moving stimuli, S1 and S2, separated by a brief delay (Fig. 1A). During each recording session, the monkeys performed the task with S1 and S2 appearing either in contralateral or ipsilateral 
A

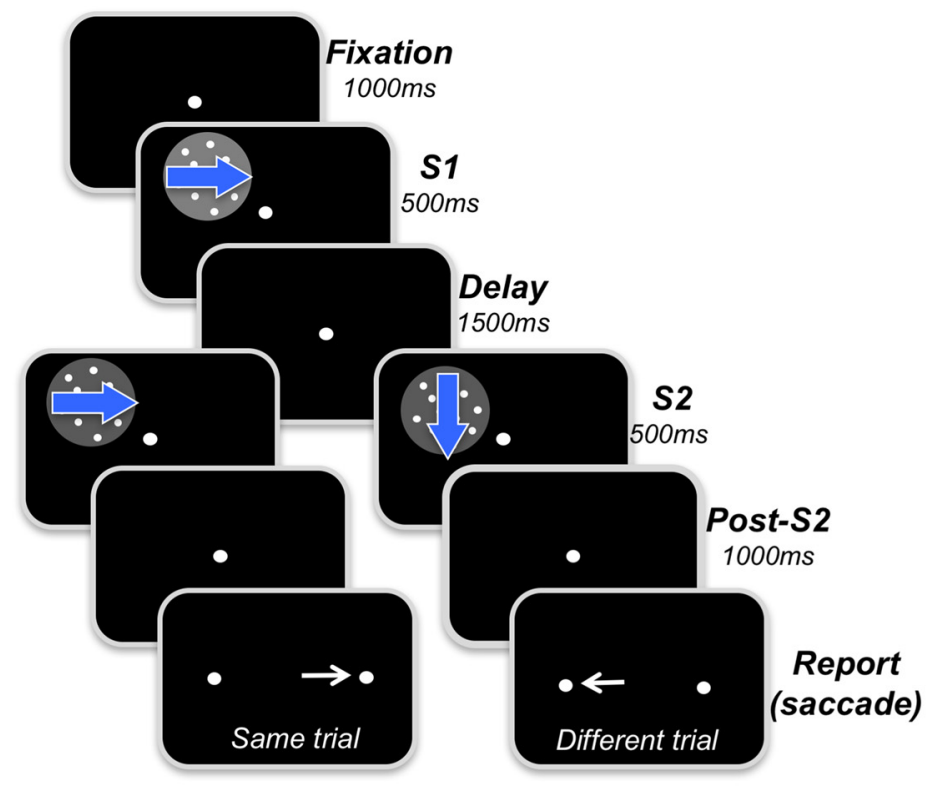

B

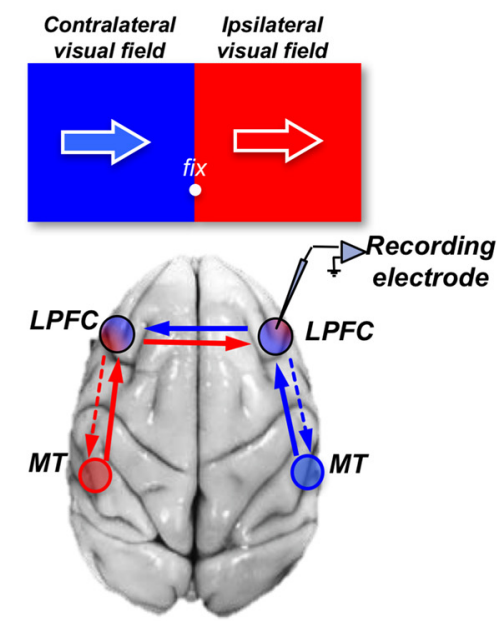

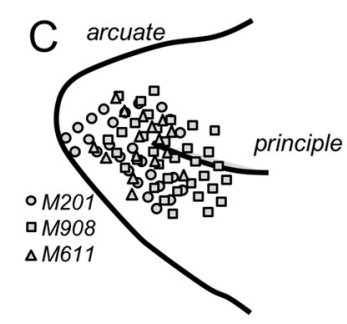

Figure 1. Behavioral tasks and recording sites. $A$, Behavioral tasks. On each trial, the monkeys viewed two directions of motion separated by a delay, $S 1$ and $S 2$, and were rewarded for reporting whether they were the same or different by making a saccade to one of two targets (right target: same; left target: different). During $\mathrm{S1}$, stimuli moved in 1 of 8 equally spaced directions and were followed by 52 stimuli moving in the same or in different directions. The animals were allowed to respond $1000 \mathrm{~ms}$ after the termination of the second stimulus (S2). B, Diagram of MT-LPFC connectivity and visual field representation in both regions. Each visual field is labeled as contralateral (blue) and ipsilateral (red) with respect to the recording electrode shown over the LPFC of the right hemisphere. The drawing shows MT providing direct input to the LPFC in the same hemisphere. Each MT is drawn as red and blue circles to indicate their representation of the opposite visual field. Solid arrows indicate bottom-up inputs from MT. Dashed arrows indicate top-down inputs from the LPFC. C, Locations of electrode penetrations for all LPFC recordings of the three monkeys (indicated by different symbols). Recording chambers were placed over the right LPFC in Monkeys 201 and 908 . Monkey M611 had recording chambers on both sides, and the recording locations were transferred to the same side.

locations in separate blocks of trials. Thus, the stimulus location was predictable within each block and all neurons were tested with stimuli appearing in each of the two hemifields. Recordings were performed in the posterior lateral portion of the prefrontal cortex, anterior to the arcuate sulcus, focusing largely on regions posterior to the principal sulcus (areas $8 \mathrm{Av}, 8 \mathrm{Ad}, 9 \mathrm{v}$, and $46 \mathrm{v}$ ) (Petrides and Pandya, 2006) (Fig. 1B). During each recording session, the performance of the three animals ranged between $80 \%$ and $95 \%$ correct and was similar for the task performed in the ipsilateral and contralateral hemifields.

The activity of two example neurons, one with an excitatory response (Fig. 2A) and one with a suppressive response (Fig. 2B), recorded during the task with stimuli placed in the contralateral and ipsilateral hemifields, illustrates both the similarities and the differences in the activity recorded with stimuli in each of the two hemifields. It also demonstrates the differences between the excitatory and suppressive cells. Responses of the excitatory cells to the ipsilateral S1 were delayed and weaker compared with responses to the contralateral S1. However, during S2, the dominance of the contralateral stimulus was reduced in one example neuron and absent in the other. Thus, the difference in responses to stimuli in the two hemifields was minimized during the more demanding comparison phase of the task. The behavior of these two neurons during S1 and S2 exemplifies the overall pattern of activity throughout the course of the trial during our recordings.

\section{Responses to contralateral motion are faster and stronger}

Because the information about contralateral stimuli is likely to arrive in the LPFC directly and about ipsilateral stimuli indirectly, we examined whether individual neurons are activated by both types of stimuli and whether the strength and the time course of their responses depend on their hemispheric origins. The analysis revealed slightly higher incidence of responses to stimuli appearing in the contralateral hemifield during $\mathrm{S} 1$ (contra, 167 of 197, 85\%; ipsi 148 of 197, 75\%; $p=0.017, \chi^{2}$ test). During the comparison stimulus S2, the proportion of neurons responding to stimuli from each of the two hemifields was nearly identical (contra, 175 of 197, 89\%; ipsi, 173 of 197, 88\%; $p=0.75$; $\chi^{2}$ test). The slightly lower incidence of responses during the ipsilateral S1 $\left(88 \%\right.$ vs $\left.75 \%, p=0.0012 ; \chi^{2}\right)$ may be a reflection of additional task demands during the comparison phase S2 (Hussar and Pasternak, 2010). 


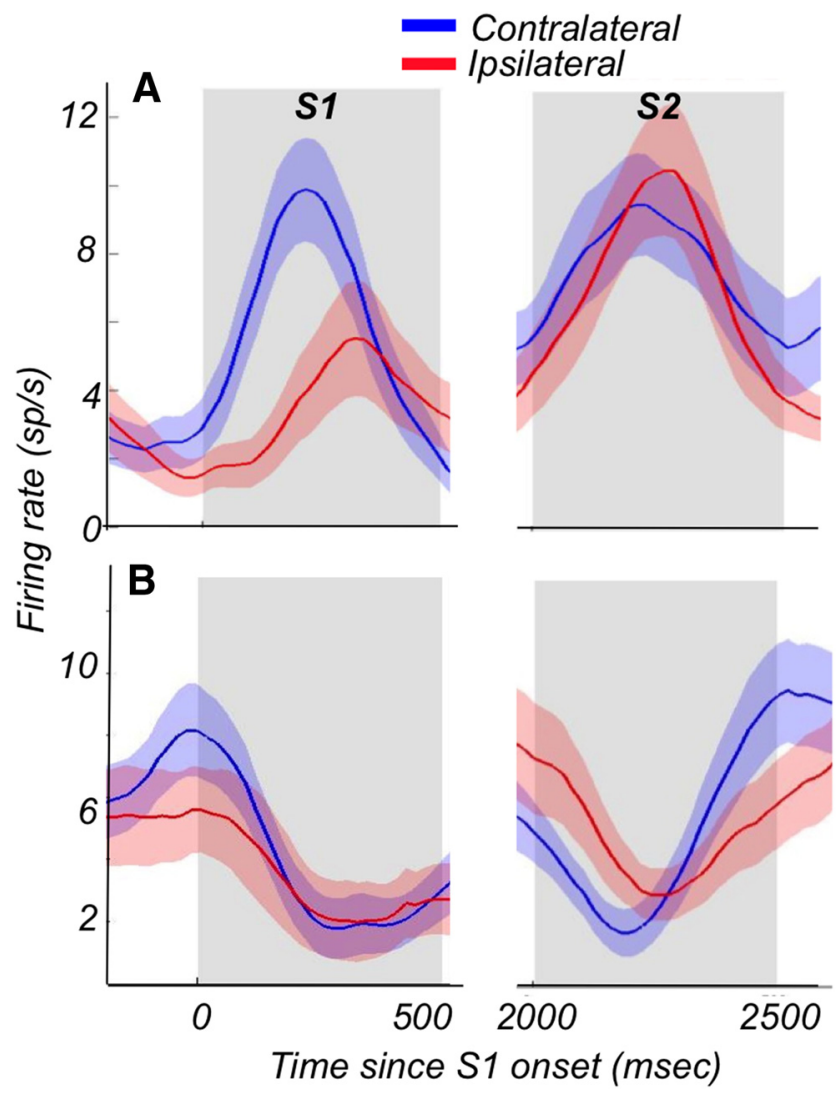

Figure 2. Activity of two example neurons on trials with contralateral and ipsilateral stimuli. Average activity for an example neuron with excitatory responses $(\boldsymbol{A})$ and suppressive responses (B). Activity of each example neurons was recorded during separate blocks of trials with both $\mathrm{S1}$ and $\mathbf{2}$ presented either in the contralateral (blue) or in the ipsilateral (red) hemifield. Only trials with responses to the preferred direction for $\mathrm{S} 1$ and $\mathrm{S} 2$ are shown.

Among neurons with reliable responses for contralateral and ipsilateral stimuli during S1 and S2, 79\% (81 of 102) were excitatory and $21 \%$ (21 of 102) were suppressive. During S1, while the excitatory responses were $\sim 20 \%$ stronger for the contralateral stimuli (Fig. $3 A ; N=81$; contra, $14.9 \pm 1.3 \mathrm{sp} / \mathrm{s}$; ipsi, $12.4 \pm 1.3$ $\mathrm{sp} / \mathrm{s} ; p=0.0031$, Wilcoxon test), suppressive responses showed no reliable difference in their firing rates for stimuli in the two hemifields (Fig. $3 B ; N=21$; contra, $-9.4 \pm 1.8 \mathrm{sp} / \mathrm{s}$; ipsi, $-9.1 \pm$ $1.8 \mathrm{sp} / \mathrm{s} ; p=0.31$, Wilcoxon test). Values of maximal firing rates listed above are not reflected in the average plotted in Figure $3 A$, $B$, because individual neurons reach maximal rates at different time points. During S2, the overall difference for the excitatory responses between the contralateral and ipsilateral stimuli was reduced and no longer significant $(N=81$, contra, $14.8 \pm 1.4$ $\mathrm{sp} / \mathrm{s}$, ipsi, $13.9 \pm 1.6 \mathrm{sp} / \mathrm{s}, p=0.22$ ). Similarly, the suppressive cells showed no difference between ipsilateral and contralateral responses $(N=21$; contra $-9.1 \pm 1.6 \mathrm{sp} / \mathrm{s}$; ipsi, $-8.7 \pm 1.5 \mathrm{sp} / \mathrm{s}$; $p=0.24)$.

The difference between responses to the contralateral and ipsilateral stimuli was also apparent in their latencies (Fig. 3C). During S1, the excitatory contralateral response emerged $40 \mathrm{~ms}$ earlier than the ipsilateral response $(N=78$; contra, $153 \pm 9 \mathrm{~ms}$; ipsi, $194 \pm 12 \mathrm{~ms} ; p=0.0004$, Wilcoxon test). During S2, the pattern was similar, although less pronounced, with contralateral excitatory responses emerging only $17 \mathrm{~ms}$ earlier $(N=77$; contra, $138 \pm 11 \mathrm{~ms}$; ipsi, $155 \pm 12 \mathrm{~ms} ; p=0.031$ ). The latency differences for suppressive responses to stimuli in the two hemifields showed the same trend, although they were not statistically sig-
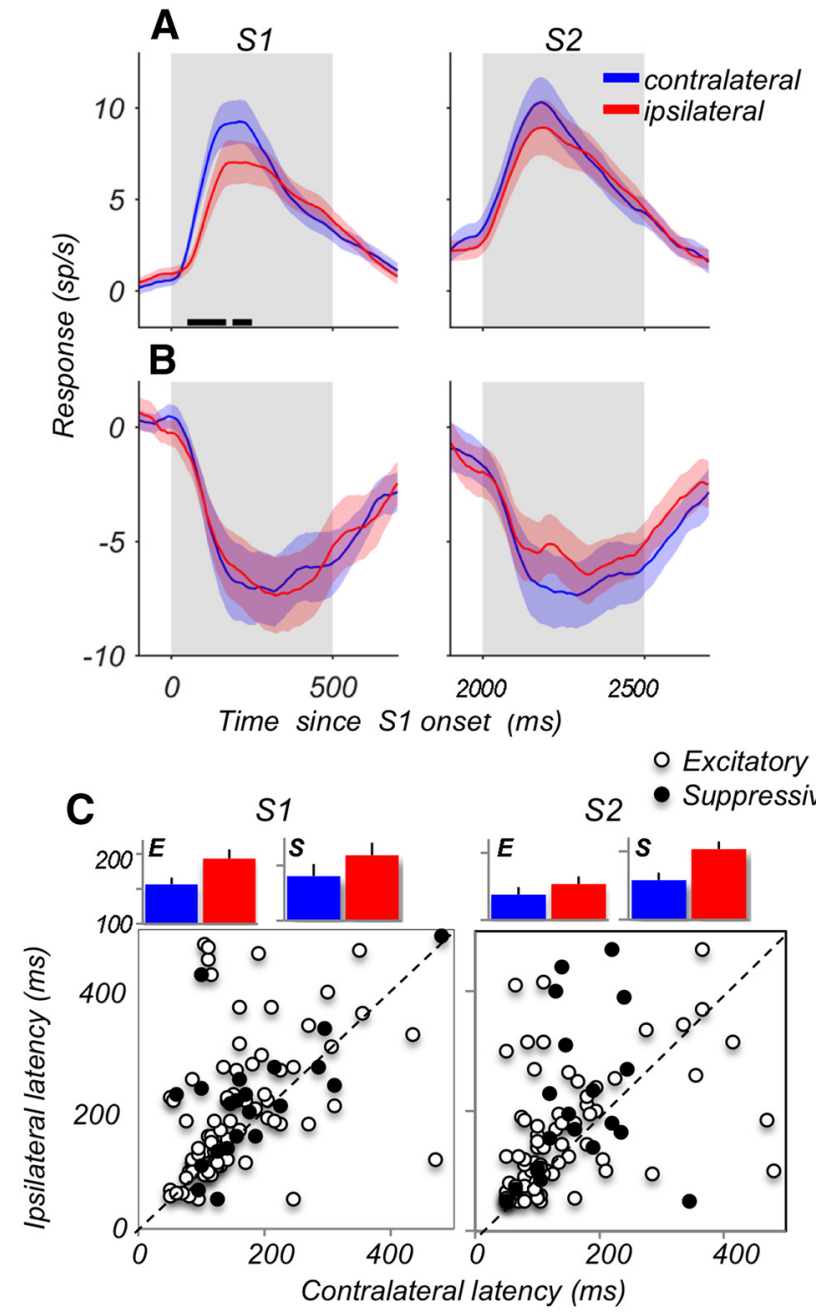

Figure 3. Responses to contralateral stimuli emerge earlier and are stronger. $\boldsymbol{A}$, Responses of excitatory cells ( $N=81$ ) during S1 and S2, for contralateral (blue) and ipsilateral (red) stimuli. The baseline activity (in a 300 ms window before the onset of S1) was subtracted for each neuron. Black horizontal lines along the $x$-axis indicate periods with significantly different responses to contralateral and ipsilateral stimuli ( $p<0.05$; permutation test). $\boldsymbol{B}$, Same as $\boldsymbol{A}$, but for suppressive neurons ( $N=21$ ). Because of the subtracted baseline, suppressive neurons show negative responses. $\boldsymbol{C}$, Comparison of latencies of contralateral and ipsilateral responses on a cell-by-cell basis. Open circles represent excitatory cells $(N=81)$. Filled circles represent suppressive cells $(N=21)$. Bar plots represent the average latencies \pm SEM for excitatory $(E)$ and suppressive $(S)$ neurons. Contralateral responses of the excitatory neurons were $41 \mathrm{~ms}$ shorter during S1 ( $p=0.0004$; Wilcoxon test) and 17 ms shorter during S2 ( $p=0.031$ ). Latencies of suppressive neurons showed the same trend, although differences between contralateral and ipsilateral responses were not statistically significant (S1: 37 ms shorter, $p=$ $0.11 ;$ S2: 45 ms shorter $p=0.11$, Wilcoxon tests).

nificant, most likely due to the lower number of neurons $(N=21$; S1: contra, $181 \pm 21 \mathrm{~ms}$; ipsi, $218 \pm 23 \mathrm{~ms} ; p=0.11$; S2: contra, $158 \pm 16 \mathrm{~ms}$; ipsi, $203 \pm 27 \mathrm{~ms} ; p=0.11$ ). Longer latencies for ipsilateral responses during $S 1$ were consistently observed in all three monkeys (M611: $32 \pm 13 \mathrm{~ms}, N=20, p=0.049$; M908: $43 \pm 15 \mathrm{~ms}, N=33, p=0.007$; M201: $40 \pm 19 \mathrm{~ms}, N=46$, $p=0.026$; Wilcoxon tests). Overall, neurons with suppressive responses had longer latencies than neurons with excitatory responses (S1: $p=0.041$, S2: $p=0.028$; Wilcoxon tests). These results highlight not only the difference between LPFC responses to the contralateral and ipsilateral stimuli but also the difference between the excitatory and suppressive neurons. The longer latencies of suppressive neurons may be a reflection of more local processing than direct bottom-up influences. It is conceivable 
that suppressive neurons constitute a functionally distinct group of cells, reflecting subsequent processing of the motion signals within the prefrontal circuitry.

In our task, the two periods of stimulus presentation differed in task requirements. During S1, stimulus direction must be identified and committed to memory. However, when the same stimulus appears during S2, the subjects not only must identify the current stimulus but also retrieve the preceding $S 1$ and compare it with the current S2. We have previously shown that, with foveal viewing, this difference in task demands during S1 and S2 was reflected in stronger responses during S2, the more demanding phase of the task, suggesting that the system may be allocating additional resources to the comparison process (Hussar and Pasternak, 2010). We examined whether the enhancement of responses during S2 observed with the foveal stimuli is also present with contralateral and ipsilateral stimuli by computing an RCI comparing firing rates of responses to the same stimuli during S1 and S2: $\mathrm{RCI}=(\mathrm{S} 2-\mathrm{S} 1) /(\mathrm{S} 2+\mathrm{S} 1)$. With this index, the value of 0 indicates identical responses during $\mathrm{S} 1$ and $\mathrm{S} 2$, the positive values $(>0)$ indicate stronger responses during S2, whereas the negative values $(<0)$ indicate weaker responses during S2. The data, shown in Figure $4 A$, reveal a higher average RCI for ipsilateral than for contralateral responses, indicating enhanced firing rates during S2 (contra: $\mathrm{RCI}=-0.030 \pm 0.02$; ipsi: $\mathrm{RCI}=+0.035 \pm 0.02 ; p=0.036, N=102$, Wilcoxon test). This response enhancement during S2 was observed only for the excitatory neurons (ipsi: $\mathrm{RCI}=0.048 \pm 0.02 ; p=0.05, N=81$, Wilcoxon test).

To track the behavior of individual neurons in response to contralateral and ipsilateral stimuli during S1 and S2, LEI = (contra - ipsi)/(contra + ipsi) was computed for each cell. In this measure, the index of 1 indicates maximal contralateral dominance, the index of -1 indicates maximal ipsilateral dominance, and the index of 0 indicates identical responses for contralateral and ipsilateral stimuli. This analysis revealed that the excitatory neurons showed strong contralateral dominance during $\mathrm{S} 1$ ( $p=$ $0.00039)$ that was not detectible during S2 $(p=0.211)$, resulting in a significant difference in the LEIs computed for S1 and S2 $(\mathrm{S} 1,0.130 \pm 0.03 ; \mathrm{S} 2,0.038 \pm 0.03 ; p=0.016$, Wilcoxon test $)$. On the other hand, responses to S1 and S2 of the less numerous suppressive cells were nearly identical (S1, $0.044 \pm 0.04 ; \mathrm{S} 2$, $0.049 \pm 0.05 ; p=0.38)$. The data in Figure $4 B$ showing LEIs for both excitatory and suppressive neurons $(N=102)$ highlight the overall contralateral dominance during $\mathrm{S} 1$ and its weakening during S2 (contra, $0.112 \pm 0.03$; ipsi, $0.040 \pm 0.03 ; p=0.034$ ).

These results suggest that individual LPFC neurons have access to both ipsilateral and contralateral information, forming relatively robust representation of visual motion from the two hemifields. The finding that many LPFC neurons enhance their ipsilateral responses during the more demanding component of the trial suggests the existence of a selective intracortical compensatory mechanism. The relative paucity of response enhancement for the contralateral stimuli represented by direct bottom-up inputs from MT supports this possibility.

\section{Similar direction selectivity for contralateral and ipsilateral motion}

We also examined whether the contralateral dominance of LPFC neurons during S1 reflected in higher firing rate and shorter latencies is also present in their selectivity for motion direction, the stimulus feature used by the monkeys to perform the task. For this analysis, we examined neurons with significant responses by comparing the incidence, time course, and strength of DS activity
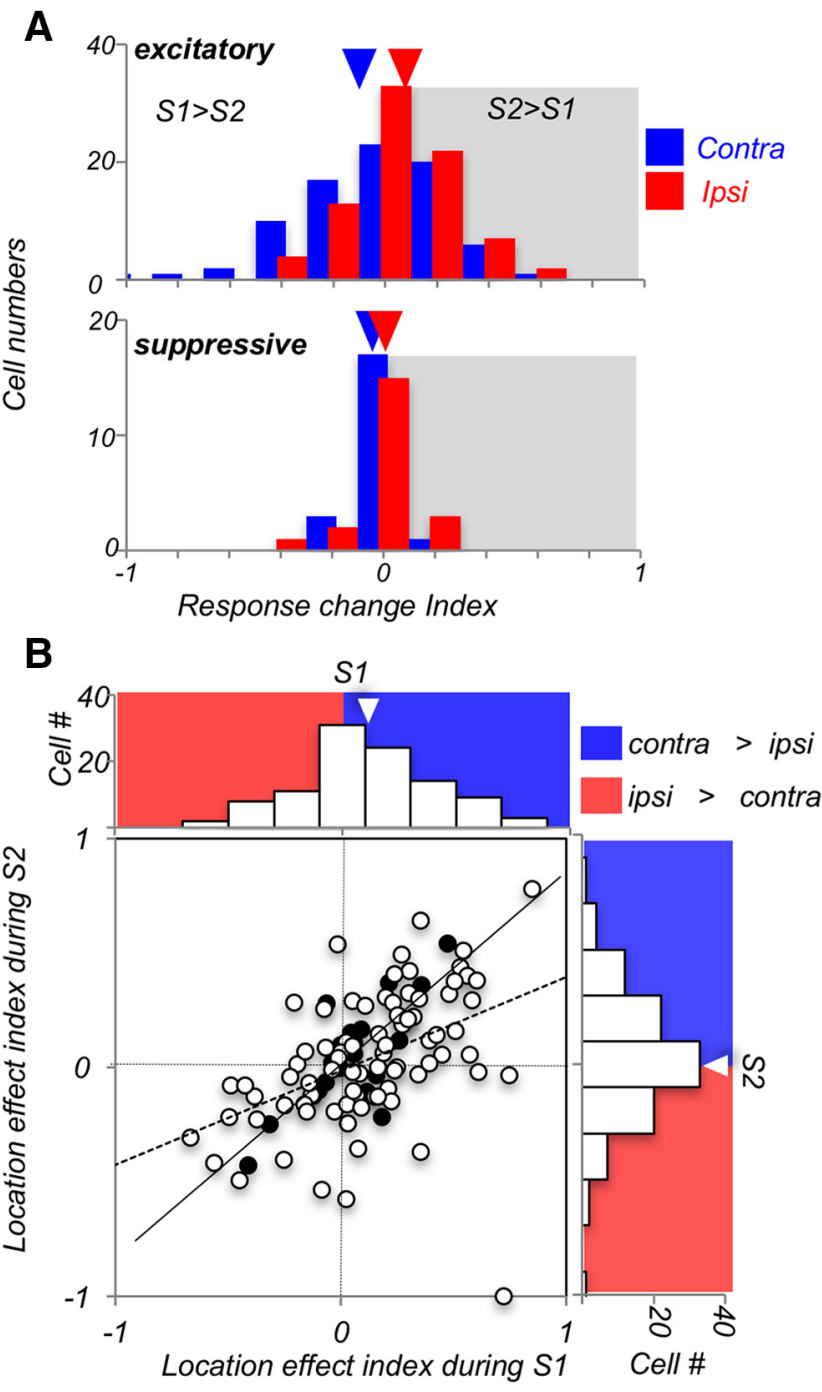

Figure 4. Ipsilateral responses are enhanced during $\mathbf{S 2}$. A, Comparison of responses during $S 1$ and $S 2 . R C l=(S 2-S 1) /(S 2+S 1)$, comparing firing rates of responses to $S 1$ and $S 2$ recorded on the same trial, for contralateral and ipsilateral stimuli. Neurons with excitatory responses (top) show, on average, only little change for contralateral stimuli, and a predominantly positive $\mathrm{RCI}$ for ipsilateral stimuli, indicating stronger $\$ 2$ responses in this condition ( $p=$ 0.018 , Wilcoxon test). Responses of suppressive neurons (bottom) show only little differences between $\mathrm{S} 1$ and $\mathrm{S} 2$, and their $\mathrm{RCl}$ for contralateral and ipsilateral stimuli is not different ( $p=$ 0.38 , Wilcoxon test). $\boldsymbol{B}, \mathrm{LEI}=$ (contra - ipsi)/(contra + ipsi), comparing the strength of contralateral and ipsilateral responses during $\mathrm{S} 1$ and $\mathrm{S} 2$ for each neuron. The dominance of contralateral responses weakens during $\$ 2$. Open circles represent excitatory cells ( $N=81$ ). Filled circles represent suppressive cells $(N=21)$. Regression lines (least-square fit to the data points) are shown for excitatory cells (solid lines) and suppressive cells (dashed lines).

for contralateral and ipsilateral motion. We used ROC analysis to identify DS activity of 102 neurons with reliable contralateral and ipsilateral responses (see Materials and Methods). We found a comparable number of neurons with reliable DS responses for stimuli in each of the two hemifields (contra, 41\%, 42 of 102; ipsi, $42 \%, 43$ of 102). This activity was equally common among excitatory and suppressive cells (excitatory, 41\%, 33 of 81; suppressive, $43 \%, 9$ of 21 ), which were combined in the following analyses. Ipsilateral and contralateral responses for preferred and antipreferred directions (with baseline subtracted) are shown in Figure $5 A, B$. Preferred direction was identified by computing the vector sum (see Materials and Methods). Although the responses were noticeably lower for the ipsilateral stimuli, their maximal 
A

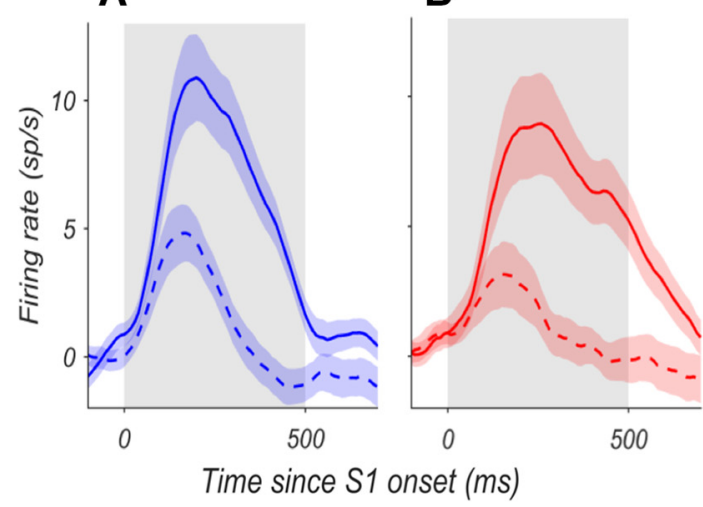

B

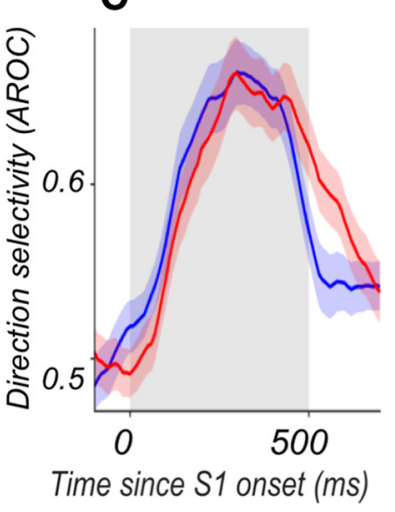

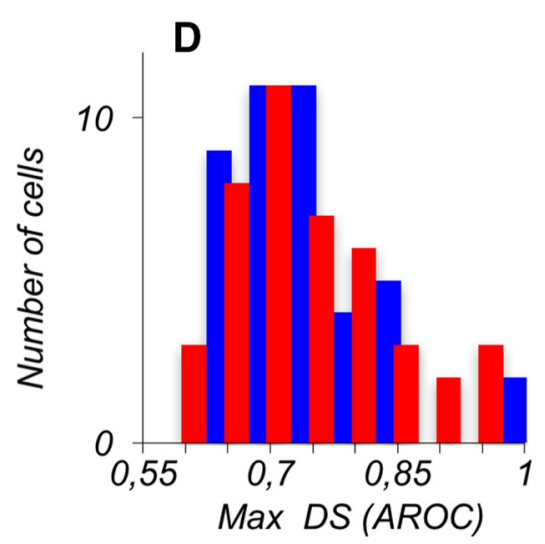

$E$
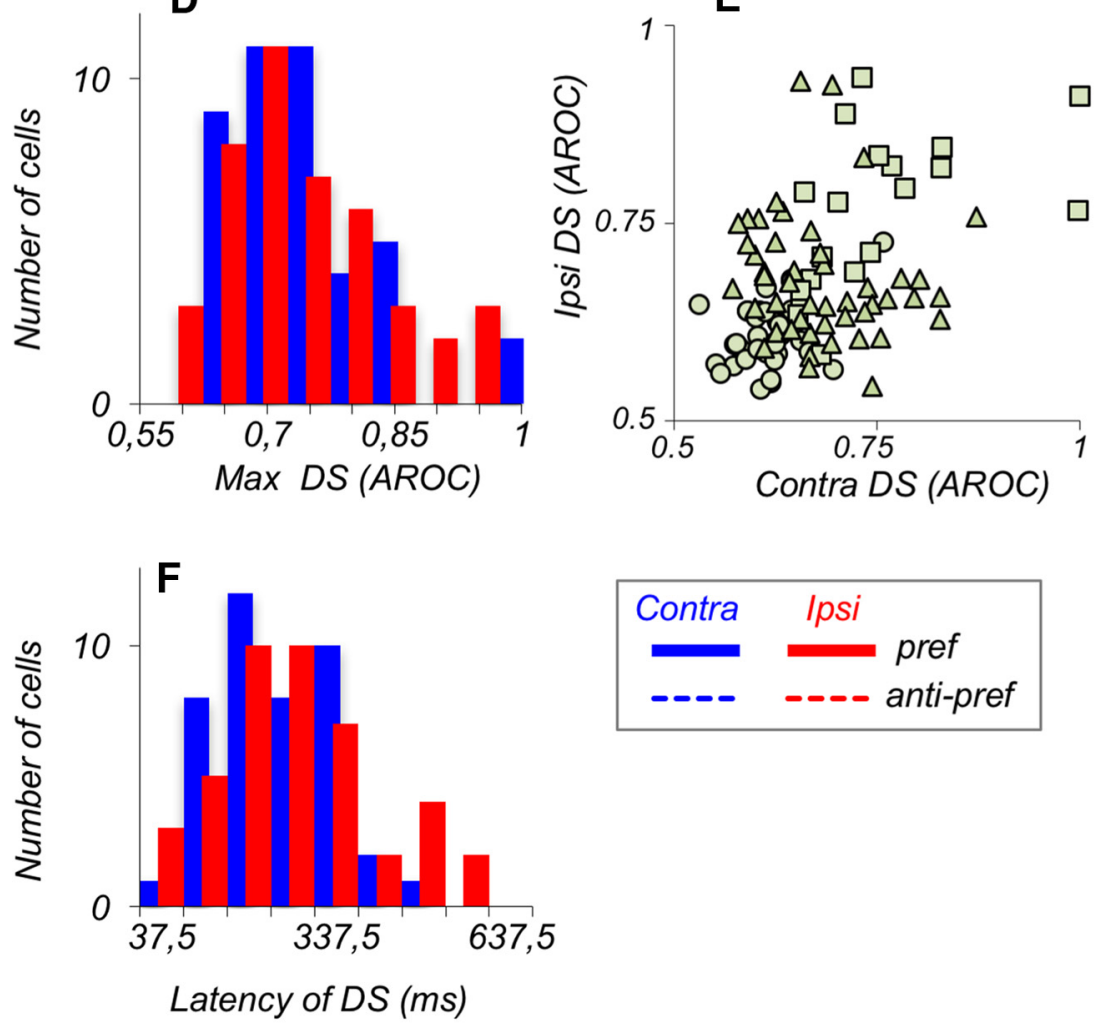

Figure 5. Contralateral and ipsilateral responses carry comparable DS signals. $A, B$, Average responses recorded during $S 1$ for preferred stimuli (solid) and antipreferred stimuli (dashed) for neurons that were DS for contralateral stimuli ( $\boldsymbol{A} ; \boldsymbol{N}=42$ of 102 neurons) and for ipsilateral stimuli ( $\boldsymbol{B} ; N=43$ of 102 neurons). The baseline activity (in a $300 \mathrm{~ms}$ window before the onset of $S 1$ ) was subtracted for each neuron, and the absolute value of the resulting firing rate was taken for suppressive neurons. $C$, Average direction selectivity of the neurons from $\boldsymbol{A}, \boldsymbol{B}$, quantified with ROC analysis (see Methods and Materials). $\boldsymbol{D}$, Distributions of maximal direction selectivity computed for ipsilateral and contralateral responses. The data for excitatory and suppressive responses were combined. There was no significant difference in selectivity for stimuli in the two locations ( $p=0.88$, Wilcoxon test). $\boldsymbol{E}$, Cell-by-cell comparison of direction selectivity for ipsilateral and contralateral stimuli. Each data point represents neurons with significant direction selectivity in both conditions $(\square)$, in only one condition $(\triangle)$, or with no significant effect $(\bigcirc)$. The correlation in the strength of DS for the two hemifields was highly significant (Pearson correlation, $r=0.443, p=3.2 \times 10^{-6}$ ). $\boldsymbol{F}$, Distribution of latencies of DS for contralateral and ipsilateral stimuli. Latencies were not significantly different (contra: $237 \pm$ $15 \mathrm{~ms} ;$ ipsi: $267 \pm 21 \mathrm{~ms} ; p=0.44$, Wilcoxon test).

selectivity for motion direction approximated that recorded with contralateral stimuli.

The strength of maximal DS quantified with ROC analysis (Fig. $5 C, D$ ), was nearly identical for contralateral and ipsilateral stimuli (contra AROC, $N=42 ; 0.742 \pm 0.01$; ipsi AROC, $N=43$, $0.740 \pm 0.01$; Wilcoxon test, $p=0.88$ ). The strength of DS for stimuli in both hemispheres was also similar in individual mon-

keys (all $p>0.1$; Wilcoxon tests). The similarity in the strength of DS for responses to ipsilateral and contralateral stimuli can be seen on a cell-by-cell basis in the scatterplot shown in Figure 5E. It shows that the strength of DS for stimuli in the two hemifields is highly correlated (Pearson correlation, $r=0.443, p=3.2 \times$ $\left.10^{-6}\right)$ : neurons with strong contralateral DS are likely to also show strong ipsilateral DS. Similarly, we found no systematic differences in the time course of these DS responses. The onset latencies of DS were broadly distributed (Fig. 5F), and average latencies of contralateral and ipsilateral DS were not significantly different (contra: $237 \pm 15 \mathrm{~ms}$; ipsi: $267 \pm 21 \mathrm{~ms} ; p=$ 0.44 , Wilcoxon test). Thus, DS responses to contralateral and ipsilateral stimuli were remarkably similar in incidence, strength, and latency.

The robust nature of DS signals reaching LPFC from the opposite hemisphere is also supported by the similarity of the tuning width for stimuli in the two hemifields. This similarity is apparent in the response of the example cell to the 8 directions of contralateral and ipsilateral motion (Fig. 6A, B). We evaluated the direction tuning by fitting a Gaussian function to the responses to ipsilateral and contralateral motion recorded during S1 $(N=102)$. The quality of the fits was evaluated by examining the $R^{2}$ values for each neuron (for details, see Materials and Methods). This analysis identified 42 neurons $(41 \%)$ with reliable direction tuning recorded with contralateral stimuli and 38 neurons $(37 \%)$ for ipsilateral stimuli (mean $R^{2}$ : contra, $0.75 \pm 0.02$; ipsi, $0.75 \pm$ $0.02 ; p=0.98$; Wilcoxon test). The average tuning curves, plotted in Figure $6 \mathrm{C}$, show the similarity of DS tuning for stimuli presented in the opposite hemifields. The bandwidths computed from individual tuning functions for each stimulus location were also similar (Fig. $6 D$; contra, $153.6 \pm$ $6.2^{\circ} ;$ ipsi, $149.9 \pm 8.4^{\circ} ; p=0.75$, Wilcoxon test).

Thus, the similarity in the strength and the bandwidth of the ipsilateral and contralateral DS suggests remarkable similarity of motion signals originating in the opposite hemispheres. We examined whether this similarity also extends to the preferences for specific motion directions displayed by individual neurons. We asked whether these preferences persist for motion appearing in opposite hemifields. Preservation of direction preferences would be consistent with nonrandom innervation of the LPFC by sensory neurons in the two hemispheres. To address this question, we examined well-tuned neurons with clearly defined preferred directions for stimuli in both hemifields (fit with a Gaussian, $R^{2}>0.5$ in both conditions; $N=21$ neurons) and found that the majority of neurons ( 14 of $21=67 \%$ ) 
A
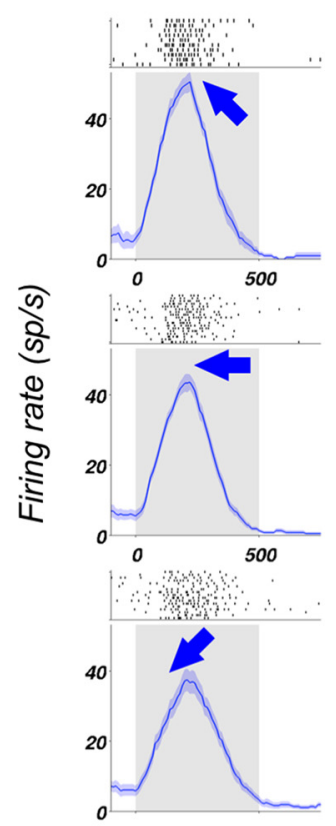

Contralateral stimulus
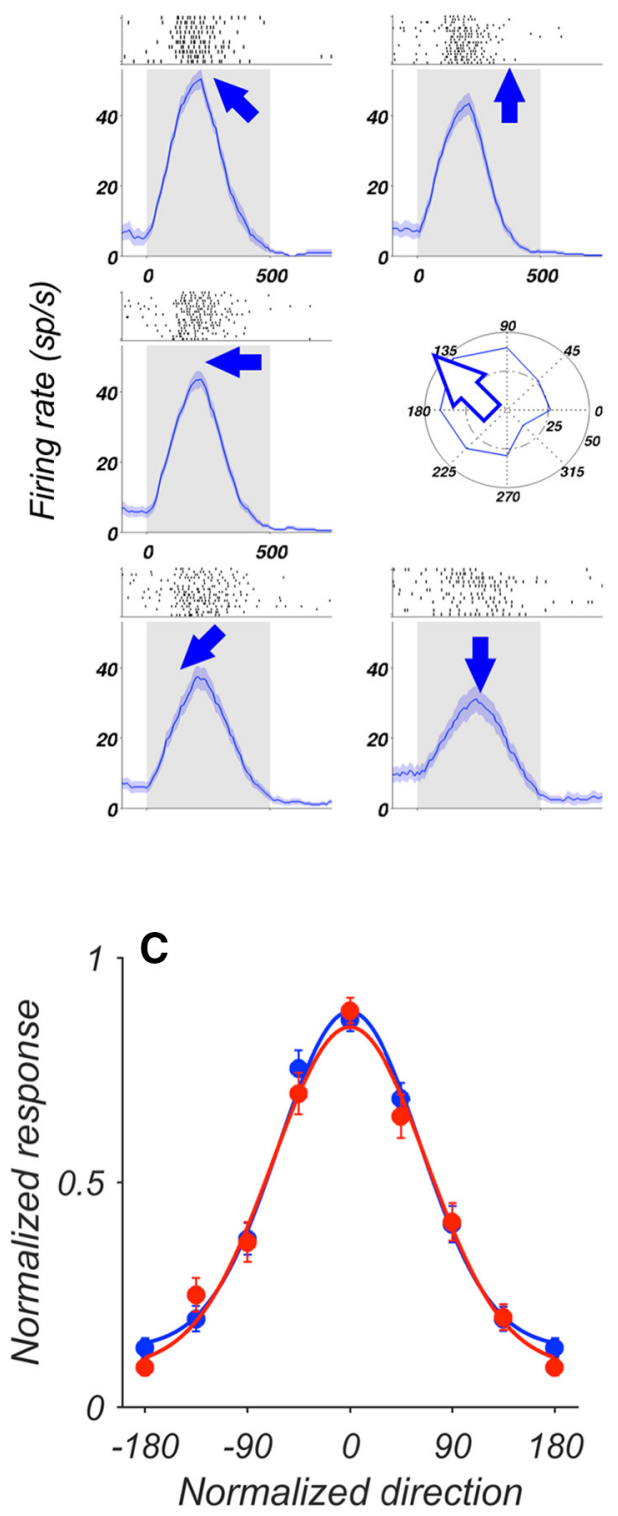
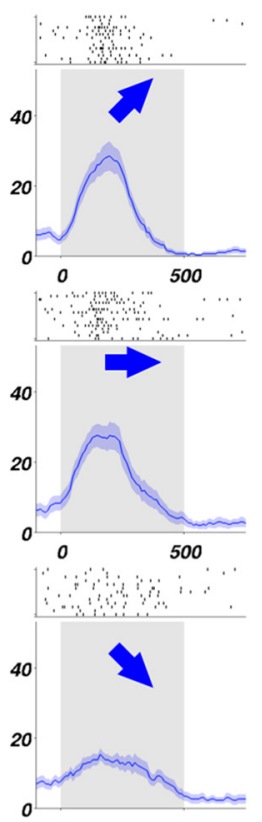

B

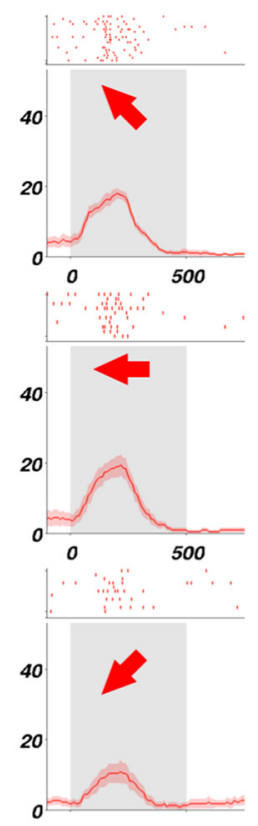

Time since $\mathrm{S} 1$ onset $(\mathrm{ms})$

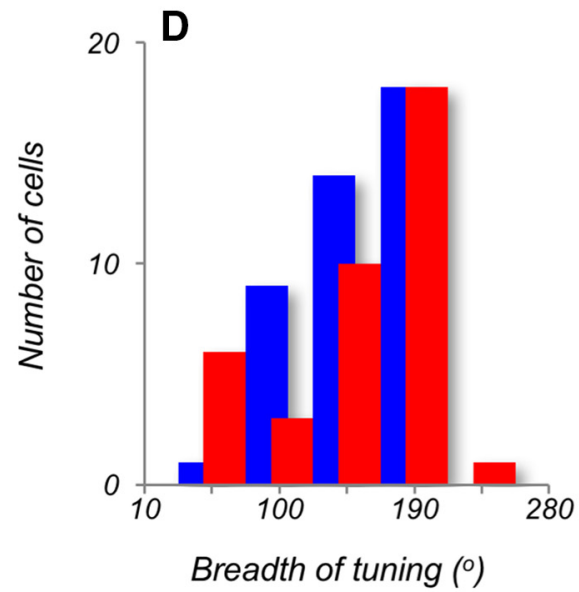

Ipsilateral stimulus
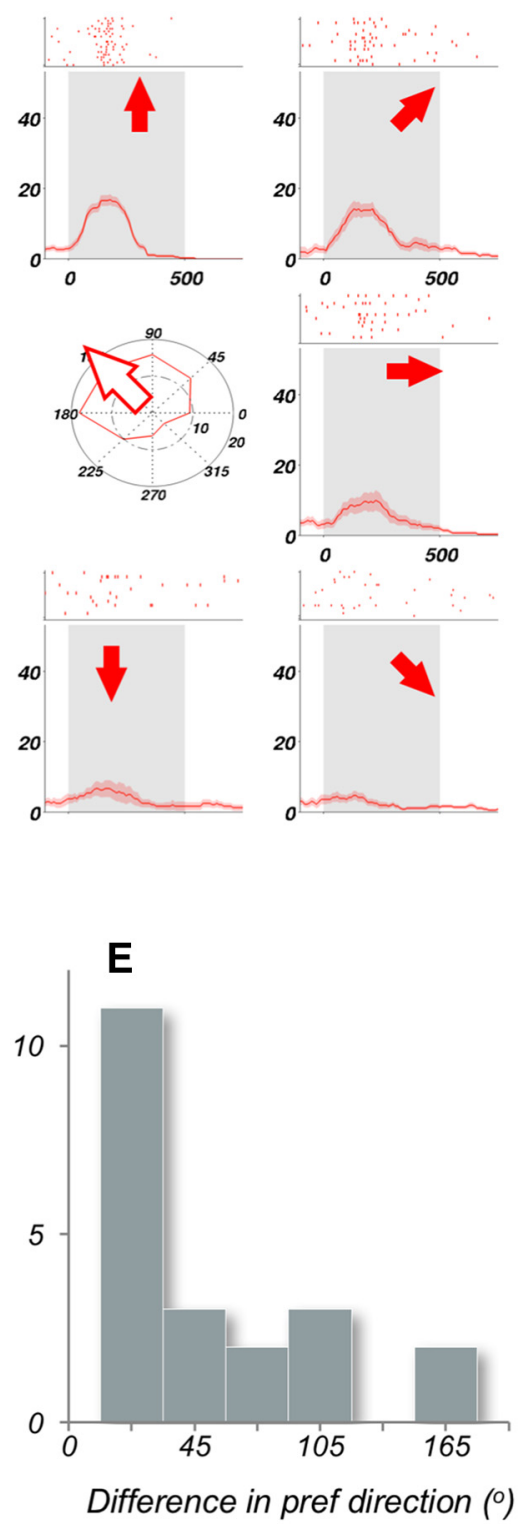

Figure 6. Similar tuning for motion direction for contralateral and ipsilateral stimuli. $\boldsymbol{A}, \boldsymbol{B}$, Direction tuning for contralateral stimuli $(\boldsymbol{A})$ and ipsilateral stimuli $(\boldsymbol{B})$ of an example neuron. Each plot represents average activity and the rasters in response to stimuli moving in eight equally spaced directions, indicated by arrows. Gray shading represents stimulus period. Polar plots in the center represent the average responses for each direction. An arrow indicates the resulting preferred direction of the cell computed using the vector sum (Materials and Methods). $\boldsymbol{C}$, Average direction tuning curve for contralateral and ipsilateral stimuli, for neurons well fit by a Gaussian (contra, $N=42$ neurons; ipsi, $N=38$ neurons; all $R^{2}>0.5$; see Materials and Methods). Individual tuning curves were aligned to $0^{\circ}$. Solid lines are a Gaussian fit to the data points. $\boldsymbol{D}$, Breadth of tuning for direction for the neurons fit by a Gaussian (same neurons as in $\boldsymbol{C}$ ). $\boldsymbol{E}$, Difference of preferred directions in response to contralateral and ipsilateral stimuli for neurons with Gaussian tuning curves $\left(R^{2}>0.5\right)$ in both hemifields $(N=21$ neurons).

showed small shifts $\left(<45^{\circ}\right)$ in the preferred direction between the two hemifields and $33 \%(N=7)$ showed a larger change in the preferred direction (Fig. $6 E$ ). Consequently, preferred directions for contralateral and ipsilateral stimuli were correlated across the population (circular correlation coefficient $\rho=0.57, p=0.011$ ) (Jammalamadaka and SenGupta, 2001). These relatively modest shifts in preferred directions suggest that LPFC may receive input from motion processing neurons in the two hemispheres, in an aligned, nonrandom manner, facilitating the integration of motion information across the visual field.

\section{Similar CEs for contralateral and ipsilateral stimuli}

Our earlier work revealed that during motion comparison tasks performed with foveal viewing, LPFC responses to the compari- son S2 were modulated by the preceding direction of S1 (Hussar and Pasternak, 2012, 2013). This modulation was in the form of enhanced responses either on trials with the same directions in S1 and S2 $(\mathrm{S}>\mathrm{D})$ or on trials with different directions in S1 and S2 $(\mathrm{D}>\mathrm{S})$. These two types of modulation, termed CEs, were represented by distinct groups of LPFC neurons and were also commonly observed in area MT (Lui and Pasternak, 2011), linking both regions to the circuitry underlying sensory comparisons. Here, to explore the nature of the potential interactions between LPFC and MT, we compared these effects for stimuli presented in the contralateral hemifield, and thus, relying on direct motion signals, to the effects measured with ipsilateral motion, conveyed to the LPFC indirectly. For this analysis, we examined neurons with reliable responses to $\mathrm{S} 2$, combining and $z$-scoring firing 
rates for trials with all 8 directions (for details, see Materials and Methods). The analysis identified 152 cells with responses during and after S2, and we compared their activity during this trial period on S-trials and D-trials (Fig. 1A). For this analysis, the activity of excitatory and suppressive neurons was combined. We used ROC analysis to identify and categorize neurons with reliable differences in responses to the two types of trials (see Materials and Methods) and found similar proportions of neurons with significant CEs for contralateral and ipsilateral stimuli (contra, $N=89$ of $152 ; 58.6 \%$; ipsi, $N=90$ of $152,59 \%$ ). For both stimulus locations, a larger proportion of cells showed preference for D-trials (contra: $60 \% \mathrm{D}>\mathrm{S}$ vs $40 \% \mathrm{~S}>\mathrm{D}, p=0.011$; ipsi: $60 \% \mathrm{D}>\mathrm{S}$ vs $40 \% \mathrm{~S}>\mathrm{D}, p=0.007 ; \chi^{2}$ tests).

Average normalized contralateral and ipsilateral responses to S2 during the $S$ - and D-trials are shown for the two populations of cells: one preferring "different" direction trials (D > S; Fig. $7 A, B$ ) and the other preferring "same" direction trials $(S>D$; Fig. $7 D, E)$. The data show striking similarity between contralateral and ipsilateral responses during the two types of trials. The more numerous $\mathrm{D}>\mathrm{S}$ group of cells showed robust and consistent difference in favor of "different" trials, which extended throughout the post-S2 period. The pattern of results was nearly identical for the CEs recorded with ipsilateral stimuli. This difference, quantified with the ROC analysis (Fig. 7C), illustrates the striking similarity between the $\mathrm{D}>\mathrm{S}$ CEs for stimuli presented in the opposite hemifields, and their similar strength (Fig. $7 \mathrm{G}$; average CE for contra, $0.706 \pm 0.01, N=53$ vs $0.696 \pm 0.01, N=54$ for ipsi; $p=0.18$, Wilcoxon test). The CEs for the less numerous $\mathrm{S}>$ $\mathrm{D}$ cells were somewhat weaker but again nearly identical for the two hemifields (Fig. $7 F, G$; average CE for contra, $0.667 \pm 0.01$, $N=36$ vs $0.668 \pm 0.01, N=36$ for ipsi; $p=0.67$, Wilcoxon test). This similarity in the strength of CEs for the two hemifields also extended to similar latencies, with a slight but nonsignificant trend for longer latencies for ipsilateral stimuli (Fig. $7 \mathrm{H}$; contra, $372 \pm 25 \mathrm{~ms}$; ipsi, $437 \pm 28 \mathrm{~ms} ; p=0.088$; Wilcoxon test). We identified a subset of 58 neurons with reliable comparison signals for stimuli in both hemifields, a majority of which consistently showed the same type of $\mathrm{CE}(\mathrm{D}>\mathrm{S}$ or $\mathrm{S}>\mathrm{D})$ in both conditions (39 of 58 neurons, $67 \%$ ). The strength of the $\mathrm{CE}$ of these consistent neurons was highly correlated across the contralateral and ipsilateral conditions (Fig. 7I; Pearson correlation, $\left.r=0.85, p<10^{-6}\right)$. These results demonstrate that CEs in the LPFC are similar for stimuli originating in either hemifield, further supporting the smooth incorporation of signals arriving from the opposite hemispheres into the activity of individual LPFC neurons.

\section{CEs depend on S1 and S2 appearing at the same location}

Reliable representation of contralateral and ipsilateral motion information in the LPFC neurons raises the question whether the comparison process recorded during S2 uses this information and/or depends on its connectivity with the motion processing neurons where it originates. We addressed this question by spatially separating S1 and S2. We reasoned that, given the robust information about contralateral and ipsilateral motion available in the LPFC, this type of manipulation would be unlikely to disrupt the comparison process, unless this process required that the two comparison stimuli stimulate the same localized receptive field, such as that characteristic of MT neurons.

To address this question, we used two versions of the task. In one, performed in two of the animals (Monkeys M908 and M201), S1 appeared in the ipsilateral and S2 in the contralateral hemifield (Fig. $8 A$; remote-ipsi). In the other version of the task, performed in one of the animals (Monkey M201), S1 and S2 appeared at the same contralateral hemifield but were vertically separated by $5^{\circ}$ (Fig. $8 B$; remote-contra). Both conditions with separated $\mathrm{S} 1$ and $\mathrm{S} 2$ resulted in a strong reduction of the CE (Fig. $8 C, D)$. This is reflected in the much lower incidence of CEs for separated S1 and S2 stimuli (same locations, $N=89$ of 152, 59\%; different locations, $N=21$ of $73,29 \% ; p=2.9 \times 10^{-5}, \chi^{2}$ test). Moreover, the great reduction in the strength of the CEs was observed across all responsive neurons in the remote-ipsi condition (AROC, $0.592 \pm 0.01, N=52$ vs same locations, AROC, $0.649 \pm 0.01, N=152 ; p<10^{-6}$, Wilcoxon test), an effect that was significant in both tested monkeys individually (Monkey M908: $p=0.03$; M201: $p=0.0007$, Wilcoxon tests). CEs in the remote-contra condition were also reduced (AROC, $0.584 \pm$ $0.01, N=21 ; p=1.8 \times 10^{-5}$, Wilcoxon test; Fig. $8 D$ ), and we found no difference in the strength of CEs between remote-ipsi and remote-contra conditions ( $p=0.98$, Wilcoxon test), reflecting a similar loss of the $\mathrm{CE}$ in both conditions. We were able to identify a subset of neurons $(N=23)$ with reliable CEs that were examined on trials with $\mathrm{S} 1$ and $\mathrm{S} 2$ appearing at the same location and also under conditions of spatial separation between S1 and S2. The data, plotted in Figure $8 E$, show on a cell-by-cell basis the loss of the CE when S1 and S2 appear at separate locations in the visual field ( $p=0.0074$, Wilcoxon test). This loss does not appear to depend on the distance between the two stimuli because it is equally pronounced with stimuli appearing in opposite hemifields, separated by at least $10^{\circ}$, as with stimuli separated by $5^{\circ}$ within the same hemifield.

We should note that spatial separation between S1 and S2 led not only to the loss of CEs but also to a reduction in the accuracy of direction discrimination (Monkey M201: same S1 and S2 locations, $79.6 \pm 0.9 \%$; remote $\mathrm{S} 1,67.0 \pm 1.3 \%$; $p=2.8 \times 10^{-6}$ Monkey M908: same locations, $86.7 \pm 0.9 \%$; remote S1, $74.0 \pm$ $1.8 \% ; p=1.8 \times 10^{-4}$, Wilcoxon tests). This parallel decrease in the accuracy of direction comparisons and in the CEs recorded in the LPFC is consistent with previously reported link between the incidence of CEs and the accuracy of direction discrimination, as well as with strong correlation between the sign and strength of CEs and the choice probability signals recorded in the LPFC (Hussar and Pasternak, 2012).

In summary, we found that the availability of relevant sensory information is not sufficient for the LPFC neurons to carry the comparison signals, arguing for the inclusion of motion processing neurons with localized receptive fields, such as neurons residing in area MT, in the comparison process.

\section{Discussion}

We found that LPFC responses to visual motion presented in contralateral and ipsilateral hemifields reflected its connectivity with motion-processing neurons. During S1, the ipsilateral responses were $\sim 20 \%$ weaker and emerged $\sim 40$ ms later than contralateral responses. However, during S2, these responses were enhanced, approaching the contralateral levels. The LPFC responses displayed remarkably similar selectivity and preferences for direction for stimuli processed in the opposite hemispheres, as well as similar signals reflecting stimulus comparison, demonstrating the seamless incorporation by the LPFC neurons of both direct and indirect sensory information. However, to generate comparison signals, LPFC neurons required that S1 and S2 appeared at the same spatial location. 

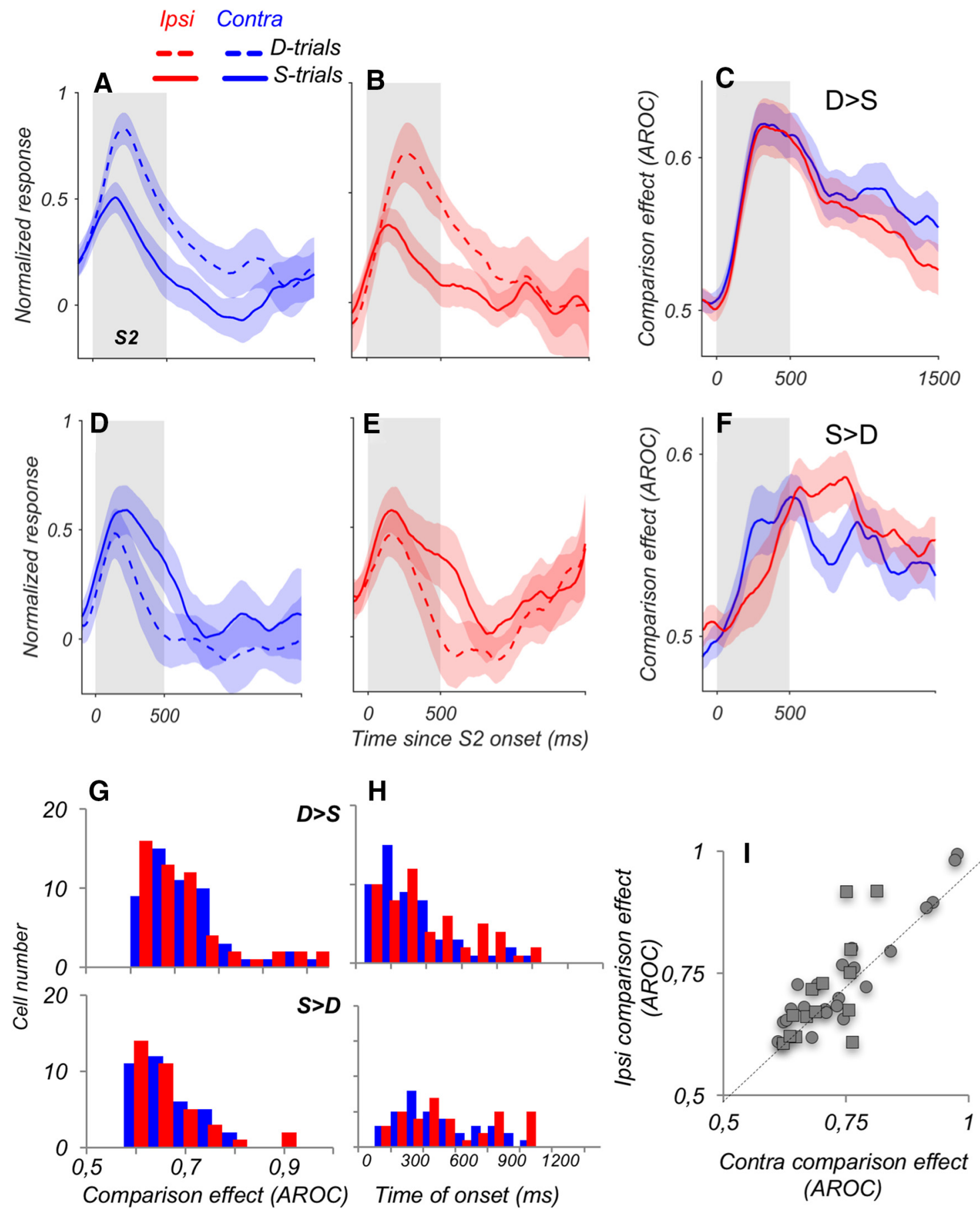

Figure 7. Similar CEs for contralateral and ipsilateral stimuli. $A, B$ Average responses of $D>S$ neurons with higher activity for trials in which the $S 2$ moved in a direction different from $S 1$ ( $D$-trials; dashed lines), shown separately for contralateral $(\boldsymbol{A})$ and ipsilateral $(\boldsymbol{B})$ stimuli (contra, $N=53 ; \mathrm{ipsi}, N=54$ ). Responses of individual neurons were normalized by subtracting the baseline activity and by dividing by the peak response during and after $S 2$. C, Average CE computed for responses during $S$ - and D-trials for $D>S$ neurons, shown in $A, B$. The difference in firing rate between $S$-trials and D-trials was quantified with ROC analysis. $\boldsymbol{D}, \boldsymbol{E}$, Average responses of $S>D$ neurons with higher activity for trials in which the $S 2$ moved in the same direction as $S 1$, shown separately for contralateral $(\boldsymbol{D})$ and ipsilateral $(\boldsymbol{E})$ stimuli (contra and ipsi, $N=36)$. F. Average $(E$ for $S>D$ neurons for stimuli in the two hemifields $(N=36)$, quantified with ROC analysis. $\mathbf{G}$, Distribution of CES for contralateral and ipsilateral stimuli, shown separately for neurons with $D>S$ effects (top) and neurons with $S>D$ effects (bottom). There was no difference in these effects for stimuli in the two hemifields ( $D>S, p=0.18 ; S>D, p=0.67$ ). $\boldsymbol{H}$, Time to the onset of (E for neurons with $\mathrm{D}>\mathrm{S}$ (top) and $\mathrm{S}>\mathrm{D}$ effects (bottom) reveals no significant differences between contra and ipsi conditions. I, Relationship of CEs for contralateral versus ipsilateral stimuli, for $D>S$ neurons $(\mathbf{O})$ and $S>D$ neurons $(\square)$. For this analysis, we selected neurons with consistent CEs in both hemifields (i.e., $\mathrm{D}>\mathrm{S}$ or $\mathrm{S}>\mathrm{D}$ in both cases; $N=39$ of 58 neurons with significant CEs in both conditions).

Contralateral responses dominate but only early in the trial sequence

Although a number of previous studies reported contralateral dominance in the LPFC (Funahashi et al., 1989; Sakagami and Niki, 1994; Rainer et al., 1998; Everling et al., 2002; Lennert and
Martinez-Trujillo, 2013; Kadohisa et al., 2015), this study provides the first quantitative assessment of ipsilateral and contralateral motion signals in individual LPFC neurons. We found that these neurons carry remarkably similar representation of stimuli appearing in the opposite hemifields. The 
A
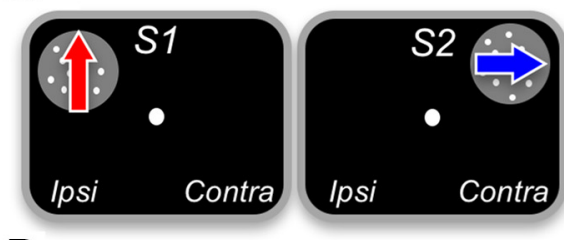

B

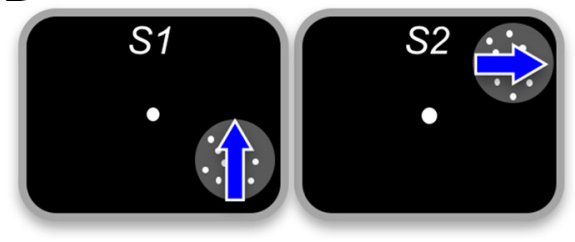

D

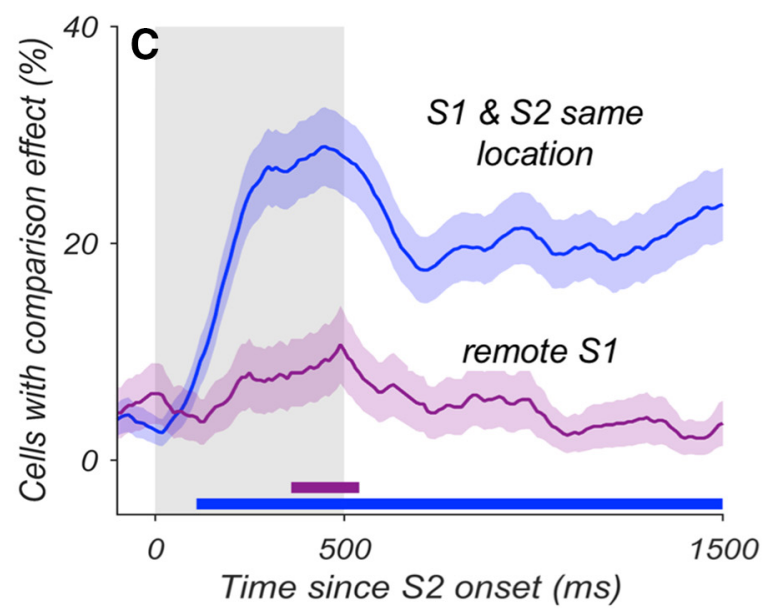

Time since S2 onset (ms)
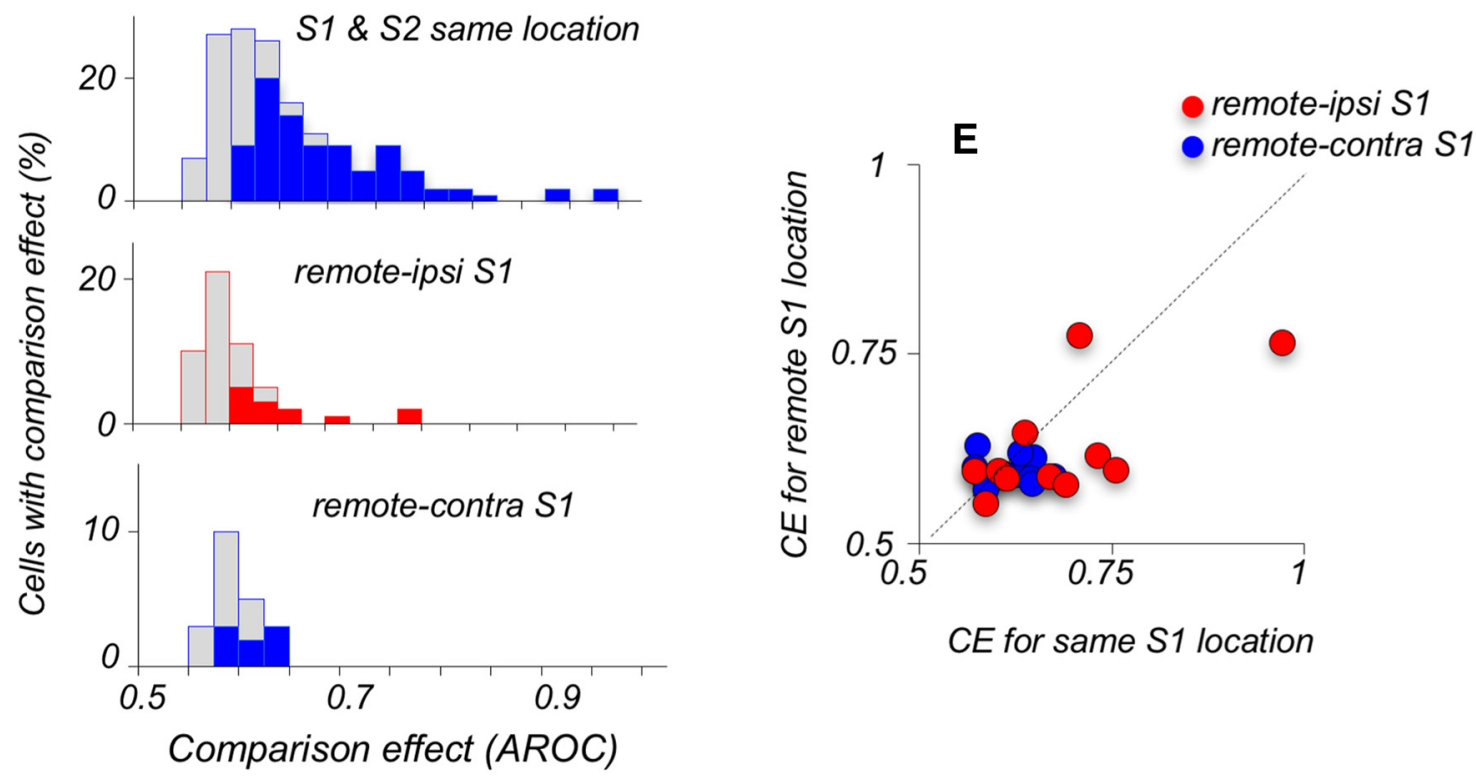

Figure 8. Comparison signals weaken when $S 1$ and $S 2$ appear at different locations. $A, B$, Diagram of stimulus locations in the tasks with separated S1 and $S 2$. S2 always appeared in the upper quadrant of the contralateral hemifield. In the remote-ipsi condition, $S 1$ appeared in the corresponding location of the ipsilateral hemifield $(\boldsymbol{A})$; and in the remote-contra condition, $S 1$ appeared in the same hemifield as $S 2$ but was displaced by $5^{\circ}$ into the lower quadrant $(\boldsymbol{B})$. In the standard version of our experiment (Fig. $\left.1 A\right)$, both S1 and $\mathrm{S} 2$ appeared at the same location. $C$, Percentage of neurons with significant $C E$ at each time point, for $S 1$ and $S 2$ at the same location ( $N=152$ neurons) and for separated $S 1$ and $S 2$ stimuli ( $N=73$ neurons). The data recorded during the two conditions shown in $A, B$ were combined. Gray shaded area represents the stimulus interval. Error bars indicate the SEMs obtained using bootstrap. Horizontal lines along the $x$-axis indicate periods where the incidence of CE is above chance level $(p<0.05$; permutation test). D, Distribution of maximum (E recorded with S1 and S2 at the same contralateral location (top), for remote-ipsi (middle), and remote-contra (bottom). Filled colored columns represent neurons with significant CE (permutation test, see Materials and Methods). Gray columns represent neurons with nonsignificant CE. The data illustrate that only very few neurons carried significant CEs when S1 and S2 were spatially separated. E, CEs for neurons that were tested with overlapping and spatially separated S1 and S2 and showed significant CEs. The data illustrate on a cell-by-cell basis that the residual CEs were greatly weakened when the comparison stimuli were spatially separated $(N=23)$.

contralateral dominance was only detectable in increased response strength and shorter latencies, consistent with their direct origins in motion processing areas MT or MST within the same hemisphere (Barbas, 1988; Boussaoud et al., 1990; Schall et al., 1995; Petrides and Pandya, 2006; Ninomiya et al., 2012). This dominance of LFPC responses based on direct intrahemispheric motion signals was most prominent during S1, whereas ipsilateral responses were enhanced during S2, resulting in a balanced stimulus representation. The observation of the late emergence of ipsilateral signals is consistent with reports of gradual transition from contralateral to ipsilateral dominance in consecutive trial epochs during an oculomotor delay task (Rao et al., 1999), as well as with the late emergence and strengthening of the ipsilateral signals in the LPFC reported for other behavioral tasks (Lennert and Martinez-Trujillo, 2013; Kadohisa et al., 2015).
In contrast, selectivity for motion direction was nearly identical in the two hemifields, attesting to the widespread availability of robust task-relevant information across both hemispheres. This similarity suggests similar motion processing mechanisms, most likely residing in MTs of the two hemispheres. Furthermore, these signals maintained remarkable consistency in their directional preferences, suggesting a high degree of coordination and alignment of signals arriving from the motion processing neurons in the two hemispheres. Although this coordination may be occurring in the LPFC, it could also be taking place at an earlier processing stage with converging MT inputs, such as area MST (Desimone and Ungerleider, 1986). Area 7a with bilateral receptive fields selective for optic flow is another possibility (Motter and Mountcastle, 1981; Heider et al., 2005). However, the alignment of preferred directions for ipsilateral and contralateral 
stimuli observed here is inconsistent with the optic flow signals encountered in area $7 \mathrm{a}$ and argue against such signals being used during our task involving localized translational motion.

We previously reported that many MT neurons show long latency, relatively weak and often suppressive DS responses to ipsilateral motion outside their classical receptive fields (Zaksas and Pasternak, 2005). We hypothesize that these responses may originate from the ipsilateral LPFC responses observed here, likely relayed via direct intrahemispheric top-down connections between LPFC and MT (Petrides and Pandya, 2006; Ninomiya et al., 2012), consistent with the recently reported information flow from the PFC to MT during a motion discrimination task (Siegel et al., 2015). This organization allows stimulus-specific top-down influences to reach sensory neurons in either hemisphere and points to a source of ipsilateral stimuli affecting direction selectivity of MT observed during feature-based attention (Martinez-Trujillo and Treue, 2004). The presence of ipsilateral responses to motion in both areas suggests that, during motion discrimination tasks, the flow of information about visual motion between LPFC and MT may be bidirectional.

\section{Comparison signals require stimuli at the same location}

As in our previous work, we found that responses of many LPFC neurons during S2 were modulated by the preceding direction in S1 (Zaksas and Pasternak, 2006; Lui and Pasternak, 2011; Hussar and Pasternak, 2012, 2013; Wimmer et al., 2016). These CEs strongly correlated with perceptual decisions and were unrelated to the largely transient stimulus-selective delay activity (Hussar and Pasternak, 2012, 2013).

Here, we asked whether these effects depend on the origins of motion signals used in their computation. We found that, as long as the two comparison stimuli appeared at the same spatial location, the incidence and the nature of CEs were nearly equivalent for contralateral and ipsilateral stimuli, suggesting remarkably balanced representation of operations relying on processing of signals originating in the two hemispheres.

The fixed mapping between trial type and report in our task (leftward saccade for D-trials and rightwards saccade for S-trials) raises a possibility that balanced representation of ipsilateral and contralateral CEs may be reflecting saccade-related activity rather than the comparison process itself. However, several factors allow us to dissociate CEs from saccade-related signals. First, the saccades reporting the trial type were only allowed $1000 \mathrm{~ms}$ after S2, long after the CEs first emerged. Second, similar CEs were observed in previous experiments in which monkeys reported their decision with button presses rather than saccades (Zaksas and Pasternak, 2006; Hussar and Pasternak, 2012, 2013; Wimmer et al., 2016). Finally, the observed loss of comparison signals on trials with spatially separated S1 and S2 makes it unlikely that CEs were associated with saccade direction.

Given the similarity of CEs for spatially overlapping S1 and S2 appearing in each hemifield, the loss of these effects on trials with separated comparison stimuli is surprising, pointing to a mechanism requiring participation of neurons with localized receptive fields residing outside the LPFC. Alternatively, if the CEs in the LPFC reflect repetition suppression (Miller et al., 1993; Woloszyn and Sheinberg, 2009) or sensory adaptation (Van Wezel and Britten, 2002; Kohn and Movshon, 2004; Mayo and Sommer, 2008; Wallish and Movshon, 2008; Solomon and Kohn, 2014), these processes would weaken or disappear with spatially nonoverlapping S1 and S2. However, this is unlikely for several reasons. First, because of stimulus-specific adaptation (Kohn and Movshon, 2004), S2 responses should decrease on "same" but not on "different" trials, inconsistent with our observations (Fig. 7). Second, although adaptation predicts a decrease in firing rates during S2, responses during contralateral S1 and S2 were similar but increased for ipsilateral S2. Third, our task structure, with a short stimulus followed by a prolonged delay, is unfavorable for strong adaptation effects (Mayo and Sommer, 2008; Wallish and Movshon, 2008). Finally, CEs in the LPFC started to emerge only toward the end of S2, making sensory adaptation in the LPFC an unlikely underlying mechanism.

A more compelling explanation of the loss of comparison signals for spatially separated S1 and S2 may be that the comparison of the current to the remembered stimulus is not performed exclusively in the LPFC and that it requires participation of neurons with localized receptive fields, such as neurons in area MT, as proposed by Hussar and Pasternak (2012). Indeed, several lines of evidence support this possibility. First, similar, albeit weaker, CEs were observed in MT (Lui and Pasternak, 2011). One difference is that in MT, the D $>$ S effect consists of two subgroups: early and late. The early D $>$ S signal is suppressive, most likely a reflection of sensory adaptation on S-trials that would be eliminated by spatial separation between $S 1$ and S2. The other two signals, late occurring $D>S$ and $S>D$, are thought to represent top-down influences arriving from the LPFC (Hussar and Pasternak, 2012). Second, we have previously shown that the precision of direction discrimination was reduced when the comparison stimuli were spatially separated and that at a given eccentricity this reduced precision occurred when the separation exceeded the distance that matched the size of MT receptive fields (Zaksas et al., 2001), at the eccentricity of $7^{\circ}$, this distance was about $4^{\circ}$, consistent with the loss of CEs with the separation of $5^{\circ} \mathrm{ob}-$ served here. Finally, there is a documented link between MT and the LPFC during the performance of the direction comparison task (Bisley and Pasternak, 2000; Pasternak et al., 2015).

We should note that the LPFC has reciprocal connections not only with area MT but also with other motion processing regions, area MST (Petrides and Pandya, 2006) and area 7a (Cavada and Goldman-Rakic, 1989). Both areas have receptive fields that are large enough to encompass spatially separated S1 and S2 (Raiguel et al., 1997) to provide LPFC with appropriate sensory signals. Nevertheless, our data suggest that such signals were largely unavailable even with the separation between the two comparison stimuli as small as $5^{\circ}$, pointing to area MT as the most likely contributor to the process.

\section{Utility of contralateral and ipsilateral signals}

Our results document balanced representation of contralateral and ipsilateral visual motion in the LPFC. Assuming that this information is used during motion discriminations, one would expect that unilateral prefrontal damage would affect discriminations of both contralateral and ipsilateral stimuli. However, there is strong evidence that such damage only affects discriminations of contralateral stimuli (Rossi et al., 2007; Voytek and Knight, 2010; Gregoriou et al., 2014; Pasternak et al., 2015). The effects of a unilateral MT/MST lesion were strikingly similar (Bisley and Pasternak, 2000), pointing to an important role of the interactions between prefrontal cortex and sensory regions representing the contralateral visual field in successful memory-guided discriminations. The 
present finding that the CEs require for the two stimuli being compared with appear at same retinal location further supports the involvement of neurons with localized receptive fields in the comparison process. In conclusion, our results suggest that, although LPFC seamlessly incorporates many key aspects of the information arriving from sensory neurons residing in opposite hemispheres, it continues relying on the interactions with these neurons at the time of generating signals leading to successful perceptual decisions.

\section{References}

Adachi Y, Osada T, Sporns O, Watanabe T, Matsui T, Miyamoto K, Miyashita Y (2012) Functional connectivity between anatomically unconnected areas is shaped by collective network-level effects in the macaque cortex. Cereb Cortex 22:1586-1592. CrossRef Medline

Barbas H (1988) Anatomic organization of basoventral and mediodorsal visual recipient prefrontal regions in the rhesus monkey. J Comp Neurol 276:313-342. CrossRef Medline

Barceló F, Suwazono S, Knight RT (2000) Prefrontal modulation of visual processing in humans. Nat Neurosci 3:399-403. CrossRef Medline

Bisley JW, Pasternak T (2000) The multiple roles of visual cortical areas MT/MST in remembering the direction of visual motion. Cereb Cortex 10:1053-1065. CrossRef Medline

Boch RA, Goldberg ME (1989) Participation of prefrontal neurons in the preparation of visually guided eye movements in the rhesus monkey. J Neurophysiol 61:1064-1084. Medline

Boussaoud D, Ungerleider LG, Desimone R (1990) Pathways for motion analysis: cortical connections of the medial superior temporal and fundus of the superior temporal visual areas in the macaque. J Comp Neurol 296:462-495. CrossRef Medline

Britten KH, Shadlen MN, Newsome WT, Movshon JA (1992) The analysis of visual motion: a comparison of neuronal and psychophysical performance. J Neurosci 12:4745-4765. Medline

Cavada C, Goldman-Rakic PS (1989) Posterior parietal cortex in rhesus monkey: II. Evidence for segregated corticocortical networks linking sensory and limbic areas with the frontal lobe. J Comp Neurol 287:422-445. CrossRef Medline

Desimone R, Ungerleider LG (1986) Multiple visual areas in the caudal superior temporal sulcus of the macaque. J Comp Neurol 248:164-189. CrossRef Medline

D'Esposito M, Postle BR (2015) The cognitive neuroscience of working memory. Annu Rev Psychol 66:115-142. CrossRef

Everling S, Tinsley CJ, Gaffan D, Duncan J (2002) Filtering of neural signals by focused attention in the monkey prefrontal cortex. Nat Neurosci 5:671-676. CrossRef Medline

Freedman DJ, Riesenhuber M, Poggio T, Miller EK (2001) Categorical representation of visual stimuli in the primate prefrontal cortex. Science 291:312-316. CrossRef Medline

Funahashi S, Bruce CJ, Goldman-Rakic PS (1989) Mnemonic coding of visual space in the monkey's dorsolateral prefrontal cortex. J Neurophysiol 61:331-349. Medline

Fuster JM (2008) The prefrontal cortex, Ed 4. London: Academic.

Gregoriou GG, Rossi AF, Ungerleider LG, Desimone R (2014) Lesions of prefrontal cortex reduce attentional modulation of neuronal responses and synchrony in V4. Nat Neurosci 17:1003-1011. CrossRef Medline

Heider B, Jandó G, Siegel RM (2005) Functional architecture of retinotopy in visual association cortex of behaving monkey. Cereb Cortex 15: 460-478. CrossRef Medline

Hussar C, Pasternak T (2010) Trial-to-trial variability of the prefrontal neurons reveals the nature of their engagement in a motion discrimination task. Proc Natl Acad Sci U S A 107:21842-21847. CrossRef Medline

Hussar CR, Pasternak T (2009) Flexibility of sensory representations in prefrontal cortex depends on cell type. Neuron 64:730-743. CrossRef Medline

Hussar CR, Pasternak T (2012) Memory-guided sensory comparisons in the prefrontal cortex: contribution of putative pyramidal cells and interneurons. J Neurosci 32:2747-2761. CrossRef Medline

Hussar CR, Pasternak T (2013) Common rules guide comparisons of speed and direction of motion in the dorsolateral prefrontal cortex. J Neurosci 33:972-986. CrossRef Medline
Jacobson S, Trojanowski JQ (1977) Prefrontal granular cortex of the rhesus monkey: II. Interhemispheric cortical afferents. Brain Res 132:235-246. CrossRef Medline

Jammalamadaka SR, SenGupta A (2001) Topics in circular statistics. Singapore: World Scientific.

Kadohisa M, Petrov P, Stokes M, Sigala N, Buckley M, Gaffan D, Kusunoki M, Duncan J (2013) Dynamic construction of a coherent attentional state in a prefrontal cell population. Neuron 80:235-246. CrossRef Medline

Kadohisa M, Kusunoki M, Petrov P, Sigala N, Buckley MJ, Gaffan D, Duncan J (2015) Spatial and temporal distribution of visual information coding in lateral prefrontal cortex. Eur J Neurosci 41:89-96. CrossRef Medline

Kohn A, Movshon JA (2004) Adaptation changes the direction tuning of macaque MT neurons. Nat Neurosci 7:764-772. CrossRef Medline

Lennert T, Martinez-Trujillo JC (2013) Prefrontal neurons of opposite spatial preference display distinct target selection dynamics. J Neurosci 33: 9520-9529. CrossRef Medline

Lui LL, Pasternak T (2011) Representation of comparison signals in cortical area MT during a delayed direction discrimination task. J Neurophysiol 106:1260-1273. CrossRef Medline

Martinez-Trujillo JC, Treue S (2004) Feature-based attention increases the selectivity of population responses in primate visual cortex. Curr Biol 14:744-751. CrossRef Medline

Mayo JP, Sommer MA (2008) Neuronal adaptation caused by sequential visual stimulation in the frontal eye field. J Neurophysiol 100:1923-1935. CrossRef Medline

Miller EK, Gochin PM, Gross CG (1993) Suppression of visual responses of neurons in inferior temporal cortex of the awake macaque by addition of a second stimulus. Brain Res 616:25-29. CrossRef Medline

Motter BC, Mountcastle VB (1981) The functional properties of the lightsensitive neurons of the posterior parietal cortex studied in waking monkeys: foveal sparing and opponent vector organization. J Neurosci 1:3-26. Medline

Ninomiya T, Sawamura H, Inoue K, Takada M (2012) Segregated pathways carrying frontally derived top-down signals to visual areas MT and V4 in macaques. J Neurosci 32:6851-6858. CrossRef Medline

Pasternak T, Lui LL, Spinelli PM (2015) Unilateral prefrontal lesions impair memory-guided comparisons of contralateral visual motion. J Neurosci 35:7095-7105. CrossRef Medline

Petrides M, Pandya DN (2006) Efferent association pathways originating in the caudal prefrontal cortex in the macaque monkey. J Comp Neurol 498:227-251. CrossRef Medline

Raiguel S, Van Hulle MM, Xiao DK, Marcar VL, Lagae L, Orban GA (1997) Size and shape of receptive fields in the medial superior temporal area (MST) of the macaque. Neuroreport 8:2803-2808. CrossRef Medline

Rainer G, Asaad WF, Miller EK (1998) Memory fields of neurons in the primate prefrontal cortex. Proc Natl Acad Sci U S A 95:15008-15013. CrossRef Medline

Rainer G, Rao SC, Miller EK (1999) Prospective coding for objects in primate prefrontal cortex. J Neurosci 19:5493-5505. Medline

Rao SG, Williams GV, Goldman-Rakic PS (1999) Isodirectional tuning of adjacent interneurons and pyramidal cells during working memory: evidence for microcolumnar organization in PFC. J Neurophysiol 81: 1903-1916. Medline

Rossi AF, Bichot NP, Desimone R, Ungerleider LG (2007) Top down attentional deficits in macaques with lesions of lateral prefrontal cortex. J Neurosci 27:11306-11314. CrossRef Medline

Sakagami M, Niki H (1994) Spatial selectivity of go/no-go neurons in monkey prefrontal cortex. Exp Brain Res 100:165-169. Medline

Schall JD, Morel A, King DJ, Bullier J (1995) Topography of visual cortex connections with frontal eye field in macaque: convergence and segregation of processing streams. J Neurosci 15:4464-4487. Medline

Schwartz ML, Goldman-Rakic PS (1984) Callosal and intrahemispheric connectivity of the prefrontal association cortex in rhesus monkey: relation between intraparietal and principal sulcal cortex. J Comp Neurol 226:403-420. CrossRef Medline

Siegel M, Buschman TJ, Miller EK (2015) Cortical information flow during flexible sensorimotor decisions. Science 348:1352-1355. CrossRef Medline 
Solomon SG, Kohn A (2014) Moving sensory adaptation beyond suppressive effects in single neurons. Curr Biol 24:R1012-R1022. CrossRef Medline

Tanji J, Hoshi E (2008) Role of the lateral prefrontal cortex in executive behavioral control. Physiol Rev 88:37-57. CrossRef Medline

Van Wezel RJ, Britten KH (2002) Motion adaptation in area MT. J Neurophysiol 88:3469-3476. CrossRef Medline

Voytek B, Knight RT (2010) Prefrontal cortex and basal ganglia contributions to visual working memory. Proc Natl Acad Sci U S A 107:1816718172. CrossRef Medline

Wallish P, Movshon JA (2008) Dynamics of neuronal adaptation in macaque MT. Washington, DC: Society for Neuroscience.

Wimmer K, Ramon M, Pasternak T, Compte A (2016) Transitions between multiband oscillatory patterns characterize memory-guided perceptual decisions in prefrontal circuits. J Neurosci 36:489-505. CrossRef Medline

Woloszyn L, Sheinberg DL (2009) Neural dynamics in inferior temporal cortex during a visual working memory task. J Neurosci 29:5494-5507. CrossRef Medline

Zaksas D, Pasternak T (2005) Area MT neurons respond to visual motion distant from their receptive fields. J Neurophysiol 94:4156-4167. CrossRef Medline

Zaksas D, Pasternak T (2006) Directional signals in the prefrontal cortex and in area MT during a working memory for visual motion task. J Neurosci 26:11726-11742. CrossRef Medline

Zaksas D, Bisley JW, Pasternak T (2001) Motion information is spatially localized in a visual working-memory task. J Neurophysiol 86:912-921. Medline 